\title{
Methodologies to Investigate Intracellular Barriers for Nucleic Acid Delivery in Non-viral Gene Therapy
}

Lotte M.P. Vermeulen ${ }^{a, b}$, Toon Brans ${ }^{a, b}$, Stefaan C. De Smedt ${ }^{a}$, Katrien Remaut ${ }^{a}$, Kevin Braeckmans $^{a, b, *}$.

*Address correspondence to Kevin.Braeckmans@UGent.be

aLab. General Biochemistry \& Physical Pharmacy, Faculty of Pharmaceutical Sciences, Ghent University, Ottergemsesteenweg 460, Ghent, Belgium

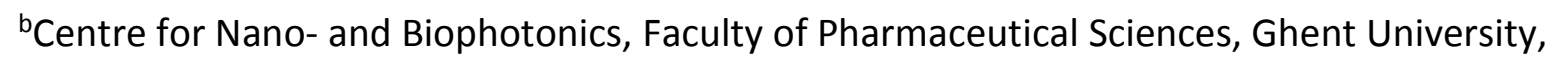
Ottergemsesteenweg 460, Ghent, Belgium 


\section{Table of Contents}

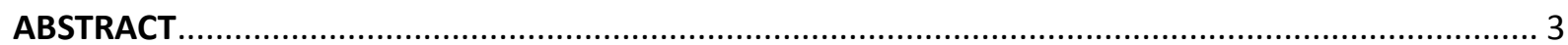

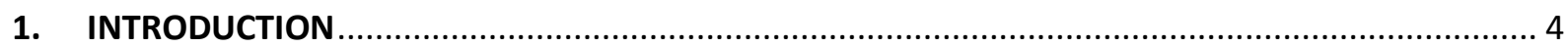

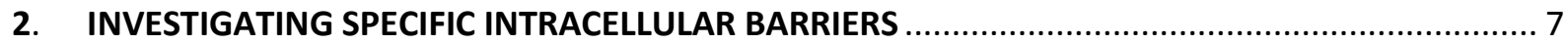

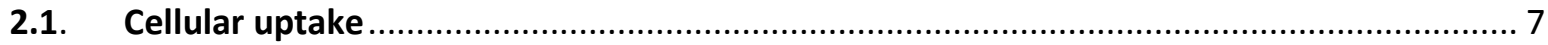

2.1.1. Evaluation and quantification of cellular uptake efficiency .................................. 8

2.1.2. Elucidating cellular uptake pathways and intracellular trafficking .......................... 16

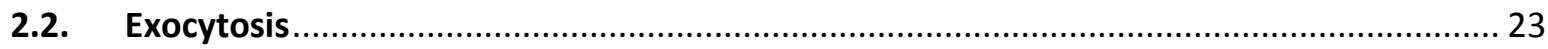

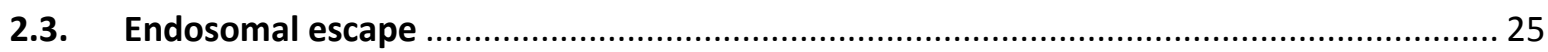

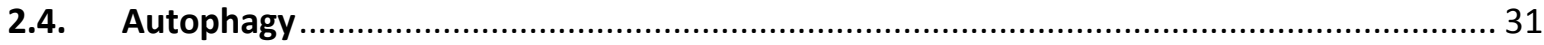

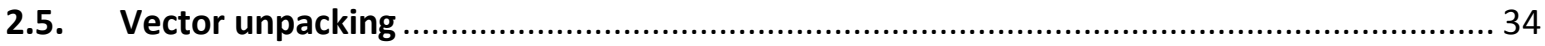

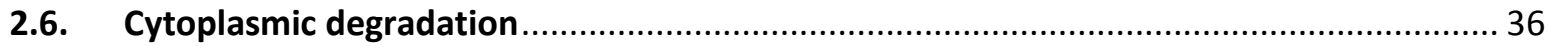

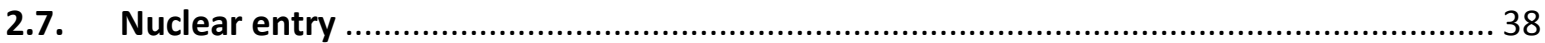

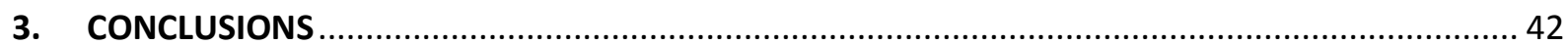

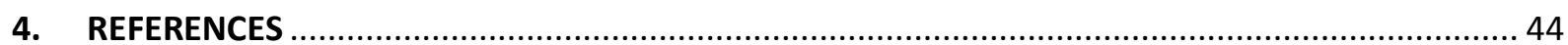




\section{ABSTRACT}

A plethora of biological barriers, intended to defend tissues and cells against external influences, stand in the way of efficient nucleic acid delivery by non-viral nanoplexes. Even when nanoplexes successfully evade extracellular barriers and reach their target cell, many intracellular barriers remain to be conquered. These include overcoming the plasma membrane, evading endosomal compartmentalization, and in some cases crossing the nuclear envelope. At the same time, exocytosis, autophagy and cytoplasmic degradation of the cargo should be avoided. Currently, there is a growing appreciation that the interaction of nanoplexes with these barriers should be understood in detail in order to rationally design a second generation of non-viral nanoplexes, capable of overcoming these many hurdles. Studying intracellular biobarriers is, however, quite challenging and specialized methods are constantly being developed. In this review, we present an overview of established as well as emerging techniques and assays that are currently available to the experimentalist to study nanoplexbarrier interaction, with a focus on quantitative methods.

\section{KEYWORDS}

Nanomaterials; Biological Barriers; Nucleic Acids; Gene Therapy; Drug Delivery; Nanomedicine 


\section{INTRODUCTION}

Gene therapy is defined as the delivery of therapeutic genes to target cells in order to replace or counteract a malfunctioning gene and has emerged as a promising strategy to cure a wide variety of inherited or acquired diseases at their genetic roots [1-4]. Besides the delivery of pDNA, several other nucleic acids (NAs), such as mRNA, siRNA and miRNA, have emerged as promising tools to modulate protein expression levels [5]. A fundamental challenge for successful delivery of these NAs lies within the engineering of delivery vectors that are both safe and effective [6]. On the one hand, viral vectors offer great efficiency in transfecting host cells, but unfortunately they suffer from several safety concerns including immunogenicity, cytotoxicity and the possibility for insertional mutagenesis. On the other hand, non-viral vectors have attracted substantial attention due to their advantages in terms of safety (reduced immunogenicity and no risk for insertional mutagenesis), lower costs, ease of production and ability to carry larger payloads [6-9]. Non-viral or synthetic vectors are typically based on cationic lipids and/or polymers that complex negatively charged NAs to form nanoplexes (NPIs) $[1,10,11]$. However, despite decades of research, the success of non-viral vectors in clinical trials remains limited due to their poor efficiency in navigating through various biological barriers[6-8].

Biological barriers for non-viral gene therapy are typically divided into two categories: extracellular (EC) and intracellular (IC) barriers. In short, EC barriers need to be overcome prior to reaching the target cell. In Figure 1, the barriers after systemic administration are shown. These include endo- and exonuclease activity in the blood circulation, interaction with blood components, activation of the immune system, surpassing endothelial barriers and migration through the extracellular space. It must be mentioned that topical administration (e.g. pulmonary and oral routes) are also intensively explored for gene therapy. Here, the EC barriers are epithelial and extra factors such as the presence of mucus and surfactant play an important role. Together, these EC obstacles can lead to cargo degradation, rapid clearance of NPIs or NPI immobilization, thereby rendering them ineffective [1,2]. Once past the EC barriers, several IC barriers obstruct the way to efficient gene delivery. First, in order to reach the IC environment, NPIs need to cross the plasma membrane $[2,12,13]$. Although physical methods, such 
as photoporation, electroporation or sonoporation, are sometimes used to provide direct access to the cell cytoplasm $[7,14,15]$, non-viral NPIs usually gain entrance to the cell by endocytosis $[5,16]$. Following endocytic uptake, NPls are localized in early endosomes which mature via late endosomes into endolysosomes. This maturation is accompanied by intraluminal acidification and activation of various degradative enzymes [5]. Hence, using endocytosis as a way to enter the cell gives rise to an additional barrier: escaping endosomal confinement before enzymatic degradation of NAs in the endolysosomes. Moreover, NPI excretion from the cell via exocytosis may happen, further reducing gene delivery efficiency [17]. Even if endosomal escape happens, the NAs (whether still complexed to the vector or not) reside in the cytosol where they have to avoid being cleared from the cell by autophagy or being degraded by cytoplasmic nucleases. Furthermore, the kinetics of cargo release from the carrier is an important consideration, since vector unpacking has been reported as another bottleneck for efficient transfection. Finally, while NAs such as siRNA, mRNA and miRNA have their site of action in the cytosol, pDNA needs to be delivered to the nucleus in order to be effective, thereby making the nuclear envelope a major barrier as well $[2,12,13,18]$.

There is a growing appreciation that understanding how non-viral NPIs behave at each of these IC barriers is necessary to rationally design more efficient NPIs [19-21]. Hence, the ability to visualize and reliably quantify the interaction of NPIs with these biological barriers is crucial [21]. However, studying IC barriers has been proven quite challenging, not in the least because of the small size and low quantity of NPIs in the cell. In this review, it is our aim to provide an overview of the entire toolbox that is available to study the different IC barriers in an in vitro setting. In this regard, we will discuss several well-established techniques, as well as emerging state-of-the-art techniques that provide exciting new possibilities. We first provide information on barrier-specific physiology and elaborate on the role these barriers play during the process of transfection with NPIs. Next, we will present assays and techniques that are available to evaluate interaction of NPIs with these barriers, including a discussion on their advantages and drawbacks. It is important to note that a considerable amount of techniques discussed in this review were developed using naked nanoparticles (NPs). Since the 
developed method is generally independent of the cargo, the methods discussed in this review are all considered to be applicable to study nanomedicines and more specific nanoplexes. Finally, we will elaborate on the gaps that remain present up to date and discuss the implementation of available techniques to boost the field in the future.

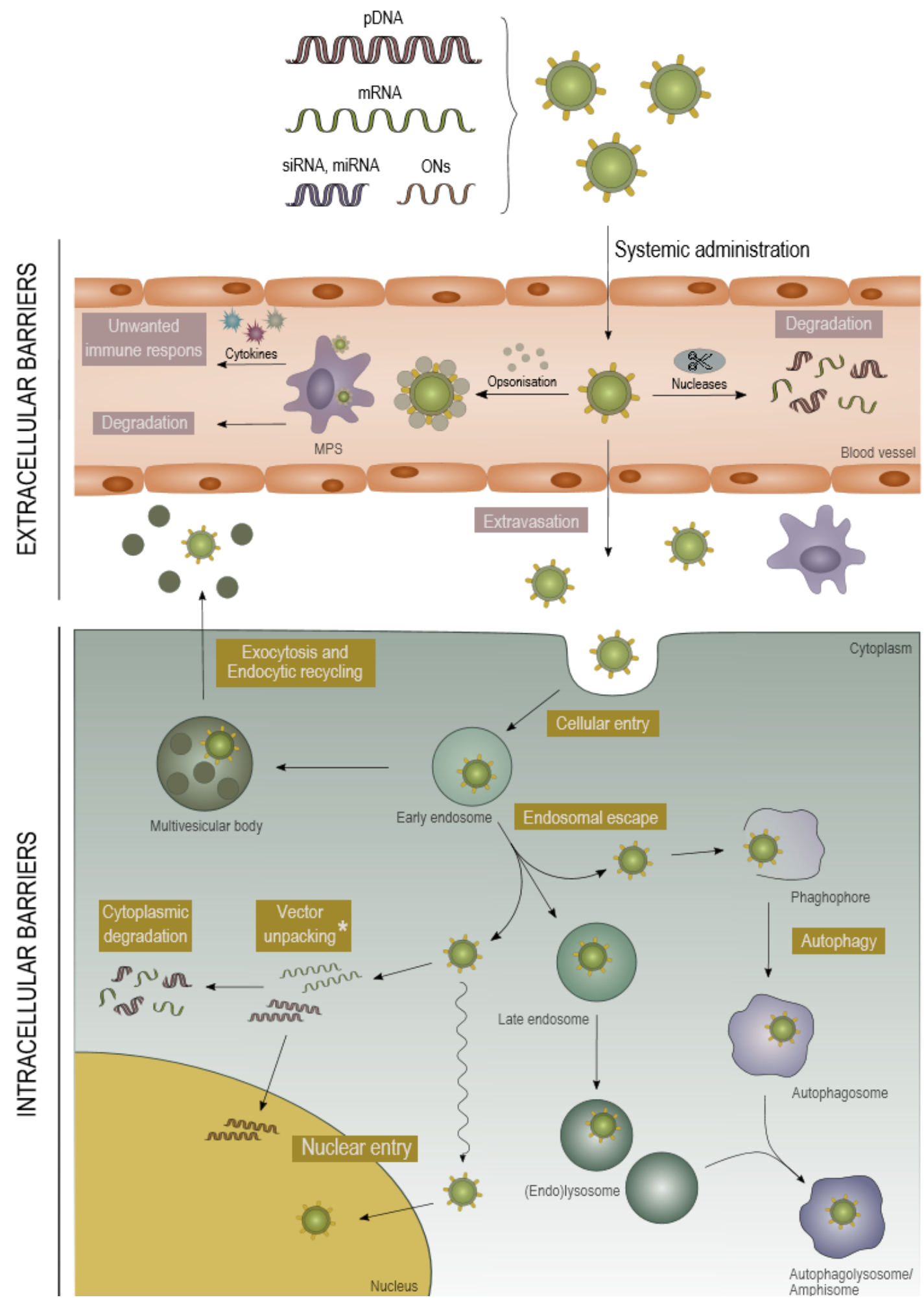


*Vector unpacking could also occur inside nucleus or endosomes.

Figure 1 Overview of extracellular (EC) and intracellular (IC) barriers for non-viral gene therapy after systemic administration. Several nucleic acids (NAs; e.g. pDNA, mRNA, siRNA, miRNA) can be incorporated in nanoparticles (NPs) in order to modulate protein expression levels. Upon systemic administration, several EC barriers are encountered: nucleases can lead to degradation of the NAs, opsonization of the NP can cause activation of the mononuclear phagocyte system (MPS) which can lead to degradation of the NPs and their cargo or to unwanted immune responses as a consequence of cytokine release. Also, extravasation poses an additional barrier for NPs to reach their target cells. Once NPs reach their target cell, there are several IC barriers standing in the way of transfection. First of all, the plasma membrane must be overcome in order to ensure cellular entry, which for NPs is usually accomplished by endocytic uptake. After endocytosis, NPs are still physically separated from the cytosol as they reside in endosomal vesicles. In order to gain access to the cytosol, the NPs must escape the endosomal pathway before the cargo is degraded in the lysosomes. Additional to lysosomal degradation, endocytosed NPs should avoid being recycled back to the EC environment via exocytosis or endocytic recycling. After reaching the cytosol, several other barriers are to be avoided including autophagy and cytoplasmic degradation. Finally, pDNA should be delivered into the nucleus, requiring passage across the nuclear envelope. Overall, vector unpacking represents an extra barrier since the cargo must be free from its vector in order to interact with its IC target. This figure was based on [6].

\section{INVESTIGATING SPECIFIC INTRACELLULAR BARRIERS}

\subsection{Cellular uptake}

Once NPIs reach their target site, they have to ensure cellular uptake by traversing the plasma membrane. The amphiphilic properties of this lipid bilayer make it an efficient barrier and gatekeeper. It provides protection to the IC components from the surrounding environment, allows to maintain homeostasis and is involved in controlling the entry and exit of nutrients and charged small molecules [22]. Although several methods have been reported for cellular uptake, the dominant mechanism for NPI cell entry is endocytosis $[5,16]$, which can be subdivided in many different endocytic pathways (as discussed in several excellent reviews [23-25]). After endocytic vesicles pinch off from the plasma membrane, they fuse with early endosomes from where the cargo is trafficked to different IC locations $[21,22]$. The understanding and quantification of cellular entry is of crucial importance, not only because efficient cellular entry is a prerequisite for effective gene delivery but also because the various endocytic pathways and subsequent vesicle trafficking have been shown to strongly affect NA delivery efficiency $[26,27]$. As there may be a link between the endocytic pathway and the final transfection efficiency, it is not only important to study the total amount of NPI uptake, but also the way they are internalized. Both will be discussed in detail in the following paragraphs. 


\subsubsection{Evaluation and quantification of cellular uptake efficiency}

Investigating cellular uptake can be carried out qualitatively (yes or no) or quantitatively (amount of uptake). Even though in some cases qualitative determination of uptake might be sufficient, quantitative determination clearly is more informative and allows to compare uptake efficiencies between cell lines or between different particles. However, before one can quantify cellular uptake, one has to think about how to characterize and quantify NPIs: by mass, surface area or particle number. All three metrics have been employed in the past, depending on experimental conditions and the method used. While mass concentration is convenient and frequently used in environmental risk assessment, surface area is considered a more suitable metric when studying interaction with biological interfaces, since these interactions are proportional to the surface area. However, quantification by particle number better reflects the particulate nature of NPIs and would, if combined with size distribution information, be the metric of choice for quantification of NPIs in biological systems [28-30]. Cellular uptake of NPIs has been studied extensively over the last decades, resulting in a large amount of methods available for evaluation of NPI internalization.

Flow cytometry allows (semi)quantification of cellular uptake in a large number of individual cells at high throughput and is therefore the most frequently used method to study NPI uptake in cells [31,32]. In this technique, size, granularity and multicolor fluorescent features of single cells passing through a light source in a fluid stream are measured $[33,34]$. In a typical experiment, cells are incubated with fluorescently labeled NPIs for a certain amount of time, after which the total fluorescence per cell is determined, which is assumed to be proportional to the number of internalized NPIs. However, several limitations should be considered when using this technique. First of all, being a non-confocal technique, flow cytometry cannot distinguish between fluorescence coming from inside the cells or fluorescence arising from NPIs that are merely attached to the outer cell surface. Yet, for NPI uptake studies, one is interested specifically in the fluorescence coming from actually internalized NPIs [35]. In order to aid in the differentiation of internalized vs membrane-attached NPIs, various strategies have been developed. The most widely used strategy is the addition of fluorescence quenchers, such 
as Trypan Blue, that can be added to the flow medium to quench extracellular fluorescence $[36,37]$. As a more specific alternative, Liu et al. developed a specific hybridization internalization probe (SHIP) assay where a fluorescent internalization probe (FIP) is incorporated into the nanoparticle (NP). Once the incubation time with the NPs is completed, a quenching probe (QP) is added. The QP hybridizes to the FIP and quenches its fluorescence on the cell surface. As the QP is unable to quench internalized FIP (no access) or other surface markers (no hybridization), fluorescence is detected only from internalized NPs (Figure 2A-B). Although the applicability of this sensor was demonstrated on the internalization of transferrin, this technique is also applicable to any type of NP with any type of cargo [38]. Another approach is to try to remove cell-bound NPIs by interfering with NPI-cell interactions or via the actual destruction of membrane-bound particles. In this regard, Braun et al. used a NP probe that comprises a fluorescently labeled polyethylene glycol (PEG) around a fluorescence-enhancing silver NP (AgNP) core. After incubation of the cells with these NPs, an etching solution is added to the cells consisting of a mixture of hexacyanoferrate (HCF) and thiosulphate (TS). HCF is used to oxidize $\mathrm{Ag}^{0}$ to $\mathrm{Ag}^{+}$, while TS clears away the newly formed $\mathrm{Ag}^{+}$ions. In this way, the core of the NP dissolves and the labeled components are released from etched AgNPs, thereby losing their fluorescence enhancement. Since both HCF and TS are charged molecules, they do not readily diffuse through cell membranes, thus protecting internalized AgNPs from being etched (Figure 2C-D) [39]. This etching technique was also reported in order to determine the cellular internalization of AuNPs and should be applicable for a myriad of NPs and cargos, as long as there is a suiting etching solution available that does not induce high levels of cellular toxicity [40]. Complete destabilization of the interaction between the NP and the cell can be realized by extensive washing with $\mathrm{NaOH}$ [41] or an acidic buffer [42]. Alternatively, when evaluating cellular uptake based on labeled cargo, polyanions (e.g. heparin) are frequently used to competitively displace the labeled NA from the carrier, followed by removal through washing [43]. Another possibility is to estimate the amount of membrane-attached NPIs via inhibition of active uptake of NPIs at $4^{\circ} \mathrm{C}$ [44]. The fluorescence measured from those cells can be used to correct the total measured fluorescence of the cells incubated at $37^{\circ} \mathrm{C}$ (having membrane-bound + internalized 
NPIs) in order to correctly estimate the fraction of internalized NPIs. One could also use non-cell penetrating reagents to achieve a double-labeling of the membrane-bound NPIs vs single-labeling of internalized NPs. In this regard, Ogris et al. labeled pDNA with YOYO-1 prior to complexation and added TOTO-3 after the addition of complexes to the cells. Since TOTO-3 also interacts with pDNA and is unable to penetrate the plasma membrane, EC pDNA is double-stained and IC pDNA is only stained with YOYO-1. Cells were then measured via flow cytometry and data were analyzed to render a ratio of fluorescence intensities on a single-cell level, which is indicative of the binding and internalization kinetics of the NPIs [45]. On the same note, Smirnov et al. incubated cells with fluorescent particles and afterwards exposed the cells to an antibody, labeled with a different fluorophore, targeted to the particles (Figure 2E). Next, using imaging flow cytometry, they quantified the number of EC (double fluorescent) and IC (single fluorescent) particles per cell, from which particle internalization was determined. Even though the authors applied this protocol to examine the binding and internalization of a pathogenic bacterium into neutrophils, it should be applicable to evaluate any cell type and NPI of interest that can be recognized using antibodies or alternatives such as streptavidin-biotin complexes [35]. As a second issue, flow cytometry uptake measurements are usually reported in arbitrary fluorescence units. However, ideally one would like to convert these values to particle numbers $[28,29]$. To this purpose, various calibration methods have been reported. For instance, Summers et al. were able to quantify the number of quantum dots (QDs) internalized per cell through calibration of flow cytometry measurements to EM data [46]. Also fluorimetry has been used to relate fluorescence intensities to NP numbers, but in this case NPs with a narrow size distribution and fixed amount of labels are needed [47]. For the quantification of internalized fluorescently labeled plasmids, flow cytometry can be calibrated with qPCR [48]. Bishop et al. transfected human primary glioblastoma cells with poly(beta-amino ester) NPIs containing Cy3 labeled eGFP pDNA. Thanks to the qPCR vs fluorescence calibration curve, the amount of $\mathrm{Cy} 3$ fluorescence could be converted to number of plasmid copies per cell [20]. A third important issue with flow cytometry has to do with the use of fluorophores to label NPIs which may alter the way in which NPIs interact with cells $[20,49]$. As such it 
may be prudent to compare results obtained with labeled and non-labeled NPIs to estimate to which extent the NPI label may influence the transfection process. Furthermore, it must be noted that the measured fluorescence intensities are not always proportionate to cellular uptake since several NPs are able to quench fluorescence, which could potentially lead to the loss of fluorescence $[50,51]$. Also, the intensity might be dependent on environmental properties, such as the $\mathrm{pH}$-dependent fluorescence of FITC fluorophores. Several fluorescent labeling strategies - with their own pros and cons - are available for NAs [52,53]. There are of course particular types of NPIs, such as NPI based on AuNPs, AgNPs or $\mathrm{TiO}_{2}$ NPs, whose uptake could be determined to some extent without the need to use fluorescent labels. Due to their strong light scattering properties uptake measurements can be based on the change in side-scattered light (SSC) [54]. 

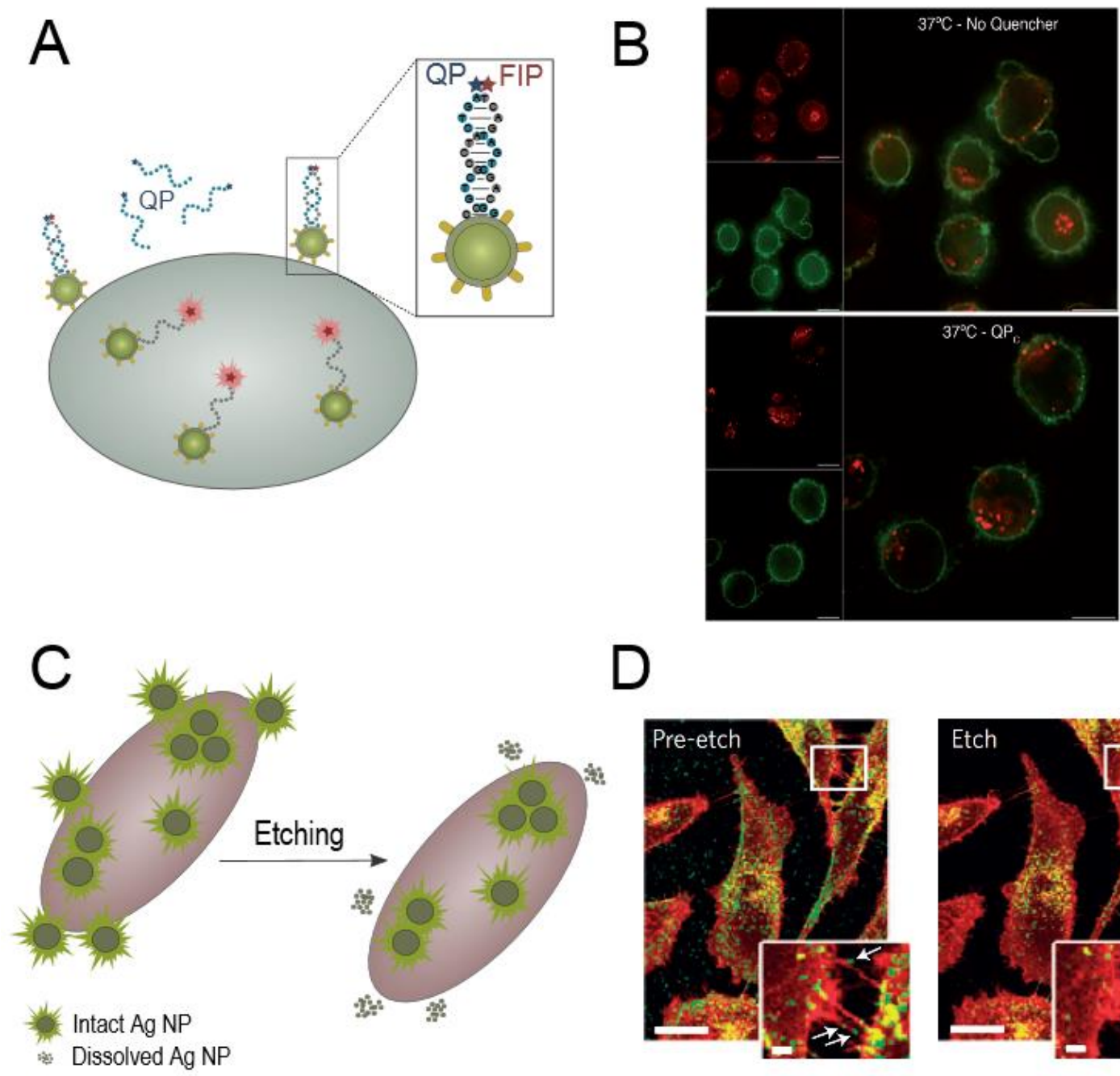

D
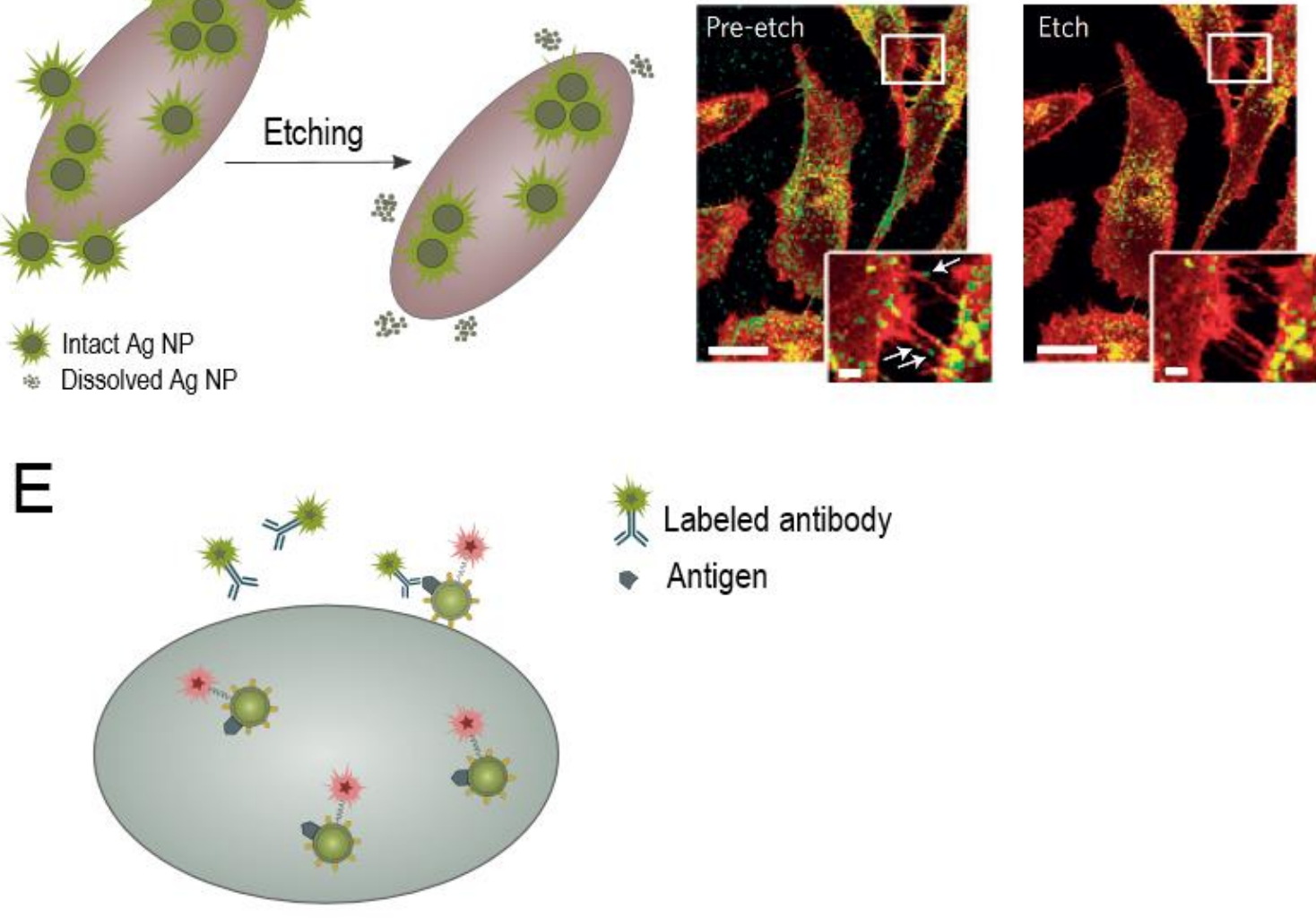

Figure 2 Strategies to distinguish between membrane-bound and internalized particles during classical uptake studies using flow cytometry. (A) Liu and coworkers developed a specific hybridization internalization probe (SHIP) to differentiate between membrane-attached and internalized NPs. The NP is labeled with a fluorescent internalization probe (FIP), which consists of a DNA strand with a fluorophore attached. Once the cells have been incubated with the NP, a quenching probe (QP) is added to the transfection medium. The QP is complementary to the sequence of the FIP, which enables it to hybridize to the FIP. Once the hybridization with the QP is completed, the fluorescence of the FIP is quenched by the QP. Since the QP is unable to migrate through the cellular membrane, fluorescence is detected only from internalized FIP. (B) Confocal images showing the efficiency of the SHIP assay. NPs are added at $37^{\circ} \mathrm{C}$ to allow active uptake of NPs, with (upper images) or without (lower images) the addition of the QP. Images A and B adapted with permission from [38]. (C 2013 WILEY-VCH Verlag GmbH \& Co. KGaA, Weinheim. (C) Schematic representation of fluorescently labeled AgNPs that are attached to the membrane and 
internalized into the cell. The brightness of the dye is enhanced by the local plasmonic field of intact AgNPs. After incubation, an etching solution is added to the cells which dissolves the Ag core of membrane-bound particles. Upon dissolution of the core, the plasmonic enhancement is lost. Internalized particles remain intact since the etching solution is unable to diffuse through the cell membrane. (D) Confocal microscopy images of cells incubated with AgNPs (green) and membrane stain (red) show how AgNPs are selectively retained in the cells after etching (right). Extracellular particles appear as green dots in the pre-etched image on the left (indicated by the white arrows) and are selectively removed after etching (right). Scalebar represents $15 \mu \mathrm{m}$ in the main images and $2 \mu \mathrm{m}$ in the insets. Images $C$ and D were adapted with permission from Springer Nature Nature Materials [39] (C) 2014 (E) This figure depicts the general mechanism, developed by Smirnov et al. with N. gonorrhoeae as a particle on neutrophils. The protocol includes the addition of labeled antibodies to non-permeabilized cells that have been previously exposed to fluorescently labeled particles. Since the antibodies are unable to penetrate into the intracellular environment, the internalized particles will be single fluorescent, while external (membrane-bound) particles will have a double-fluorescent labeling.

A second well-established technique to evaluate NPI uptake inside cells that is often used in conjunction to flow cytometry is light and fluorescence microscopy. This technique has proven to be extremely useful to study NPI distribution and qualitative uptake [55-58] and is also - to a minor extent - used to quantify cellular uptake of NPIs $[59,60]$. Quantification of NPI uptake via light microscopy is, however, not that straightforward. While the total fluorescence per cell could be quantified if cells can be segmented reliably in the images, optical microscopy does not possess the necessary resolving power to detect single NPIs, a key requirement for the quantification of particle numbers $[28,61]$. Recently, so-called "super-resolution" microscopy techniques have emerged that go beyond the diffraction limit and reach a resolution of $<100 \mathrm{~nm}$. Van der Zwaag et al. reported on the use of stochastic reconstruction microscopy (STORM) to extract quantitative information about the size, number and positioning of ovalbumin-loaded polystyrene NPs inside HeLa cells, while Peuschel et al. used stimulated emission depletion microscopy (STED) to quantify the uptake of unloaded silica NPs in A549 cells [26,62]. These promising results confirm that super-resolution microscopy can be used successfully in the quantification of cellular uptake on a single particle level.

Due to the small de Broglie wavelength of accelerated electrons, electron microscopy (EM) provides superior spatial resolution ( $<0.2 \mathrm{~nm}$ for TEM; $\pm 1 \mathrm{~nm}$ for SEM) and is the technique of choice when it comes to resolving structures that are below the optical resolution limit $[22,63,64]$. Thus, EM is frequently used as a third technique to evaluate [65-69] or quantify [70-72] cellular uptake. RothenRutishauser et al. compared uptake of AuNPs in A549 cells via CLSM and TEM. AuNPs can be easily detected by TEM because of their high electron density and by CLSM due to the addition of the red 
fluorescent marker ATTO 590. Quantification via TEM resulted in the total number of internalized NPs since TEM, in contrast to CLSM, provides enough spatial resolution for detection of single NPs [73]. In order to downsize the complicated and time-consuming sample preparation that accompanies EM, liquid EM is regarded as an emerging field since it can eliminate some of the steps that are required for sample preparation in classic EM [74]. Peckys and de Jonge used a liquid-STEM approach to study live fibroblast cells with AuNPs using a microfluidic chamber. Since their approach can be used to study whole cells with a thickness up to $\pm 10 \mu \mathrm{m}$, the need for sectioning is excluded and only minimal sample preparation is required [75]. In 2014, Peckys and de Jonge performed imaging of whole cells in a fully hydrated state using environmental scanning electron microscopy (ESEM), resulting in a sample preparation time that is comparable to that for light microscopy [76]. Alternatively, volume restrictions may be eliminated by the use of focused ion beam scanning electron microscopy (FIB-SEM), which continuously sections matrix-embedded cells using an ion beam, thereby making 3D SEM images possible $[54,72]$. Apart from volume restrictions and time-consuming sample preparation, another issue that is often encountered in EM imaging is low contrast. Indeed, the NPI of interest can only be seen if the carrier has sufficient contrast compared to the biological material. Inorganic NPs like gold NPs and superparamagnetic iron oxide NPs can be distinguished easily, but organic NPs usually need contrast enhancing agents in order to be visualized [77].

Among the analytical techniques, one could determine cellular uptake by measuring the fluorescence intensity of a cell-lysate after incubation with fluorescently-labeled NPIs [78]. However, since this technique also does not discriminate between membrane-bound and internalized NPIs, one should find a way to distinguish the two forms, as discussed above. Furthermore, this technique provides information on a population level instead of on a single-cell level. Alternatively, inductively coupled plasma mass spectrometry (ICP-MS) methods are frequently used to quantify cellular uptake of NPs and NPIs [79-82]. In ICP-MS the sample is ionized and its elemental composition is analyzed via MS. Alternatively, the ionized sample can be analyzed by atomic emission spectrometry (ICP-AES), also referred to as optical emission spectrometry (ICP-OES) $[29,83,84]$. Both methods can be used for high- 
throughput analysis of a wide range of NPs such as metals, (metal)oxides and quantum dots. The main benefits of ICP-MS over ICP-OES/AES is the higher sensitivity of ICP-MS (ppt range) compared to ICPOES/AES (ppm range) and that ICP-MS is capable of providing isotope information $[28,29,61]$. An important limitation of these techniques is that they do not allow quantification of NP uptake on a single cell level as the analysis is performed on the cell population as a whole. It is however possible to extrapolate this data to calculate the average NP uptake per cell after determination of the total number of cells per sample. Nevertheless, one must take into account that this average is expressed as the average mass of the element per cell instead of the average number of NPIs per cell, although a conversion is possible if the mass and size of the NPIs is known $[29,85]$. Modifications of ICP-MS have become available that allow single cell ICP-MS [86] or single particle ICP-MS [87]. In both cases, highly diluted samples are used so that each signal corresponds to a single particle or cell. Single particle ICPMS allows quantification of mass concentration, particle size and particle number in complex environmental and biological matrices, while single cell ICP-MS allows quantification of NPI content in individual cells $[85,87,88]$. A second issue that needs to be overcome is, similar to flow cytometry, the differentiation between cell-associated NPIs and internalized NPIs. As these methods are often used on metallic/metal oxide NPs, chemical etching is typically used as discussed above and schematically presented in Figure $\mathbf{2 C}$ [40]. As a possible alternative, Hou et al. described a rapid laser-based method to selectively desorb and ionize extracellular AuNPs [89].

Other techniques to study NP uptake - often less frequently used - include Raman spectroscopy and labeling of particles with radioisotopes. In Raman spectroscopy, a Raman fingerprint is obtained based on inelastic scattering of laser light due to the interaction of photons with molecular vibrations. Inelastic scattering of photons is a very inefficient process, but Raman band intensities can be enhanced several orders of magnitude by adsorbing a molecule on a metallic surface, typically composed of Au or Ag $[90,91]$. Surface enhanced Raman scattering (SERS) offers high sensitivity, minimal sample preparation and resistance to photobleaching and was successfully applied for the quantification of NPs uptake $[92,93]$. Huang et al. used SERS to investigate the cellular internalization 
of graphene oxide loaded with gold NPs. By combining SERS with light microscopy and TEM, they concluded that cell entry happened mainly via energy-dependent, clathrin-mediated endocytosis [94]. Furthermore, it should be mentioned that labeling of NPIs with radioisotopes has also been used to quantify NPI uptake in cells. This approach allows quantitative evaluation with high sensitivity both in vitro and in vivo $[95,96]$. However, many drawbacks are related to working with radioisotopes such as difficult particle preparation and restricted use by regulations involving disposal of materials [28]. On a side note, it is worth mentioning that PCR is frequently used to quantify the amount of NA molecules delivered to the IC environment. Colombo et al. quantified intracellular siRNA concentrations using reverse transcription (RT) and stem-loop quantitative PCR after administration of siRNA-loaded lipidpolymer hybrid NPIs to H1299 cells that stably expressed eGFP [97]. The technique has a wide range of quantification but lysing cells, isolating and purifying DNA and running PCR is considered a timeconsuming process. However, some researchers state that it should be amenable to high-throughput formats and automation $[19,20,98,99]$.

\subsubsection{Elucidating cellular uptake pathways and intracellular trafficking}

NPIs commonly find their way into cells through distinct endocytic pathways, as reviewed elsewhere [23-25]. A common methodology to find out which pathways are involved is via exclusion studies where pharmacological or biological agents that can shut down a particular pathway are used and any remaining NPI uptake is quantified. The decrease in NPI uptake relative to untreated cells is a measure for the importance of that particular pathway $[25,100]$. While any of the above mentioned techniques to measure NPI uptake can be used for this, in practice flow cytometry is used the most. A plethora of pharmacological inhibitors is available $[23,101]$ and their use is quite widespread $[100,102-104]$. Yet, one should bear in mind that such inhibitor experiments should be interpreted with care. It has been shown that chemical inhibitors of endocytosis, such as chlorpromazine or genistein, show poor specificity and that their effect is highly cell line-dependent [104]. To avoid the problem of nonspecificity, biological strategies such as RNA interference or the introduction of dominant-negative mutants can be used to inhibit specific endocytic pathways $[101,105,106]$. The main drawback here is 
that these compounds could affect cell viability and are frequently observed to influence other endocytic pathways $[101,104]$.

Fluorescence colocalization microscopy is another very popular technique to study not only internalization pathways but especially the subsequent intracellular trafficking of NPIs. Here, colocalization analysis methods are applied to microscopy images of fluorescent NPIs that may or may not coincide with fluorescently labeled subcellular structures or compounds [107-109]. Labeling of specific endocytic proteins is frequently performed either by immunostaining of fixed cells or, in live cells, by transfecting cells with fluorescent protein constructs (Figure 3A) [110]. Specific proteins related to uptake and trafficking include caveolin-1, flotillin-1, Rab5, Rab7 and LAMP-1, markers for caveolae-mediated endocytosis, flotillin-mediated endocytosis, early endosomes, late endosomes and lysosomes respectively. However, one should be mindful of potential artifacts due to protein overexpression, such as interactions that do not occur under normal circumstances due to low affinity, miscolocalization of the fusion protein or aggregation and fusion of organelles [100]. One notorious example is the mistaken discovery of the so-called "caveosome", which was later declared to correspond to late endosomal compartments modified by the accumulation of overexpressed caveolin-1 awaiting degradation instead of a distinct endosomal compartment [111,112]. Apart from labeling specific proteins, a diverse array of organelle probes is available such as Lysotracker (lysosomes) or Mitotracker (mitochondria) that are designed to accumulate in specific IC structures (Figure 3B) [113,114]. Using Lysosensor yellow/blue, Wang et al. performed ratiometric imaging to study the effect of cell-penetrating peptides on the intracellular distribution of NPs in live cells. The Iblue $/$ green ratio of Lysosensor in every pixel was measured and assigned a $\mathrm{pH}$ value based on a calibration curve. These $\mathrm{pH}$ values were then categorized into endosomes $(\mathrm{pH} 5-6)$ or lysosomes $(\mathrm{pH}$ $<5)$ and distribution of NPs in endolysosomal compartments was quantified by correlating the pH value with the fluorescence intensity of the NP at each pixel [115]. It should be noted however that combining acidotropic dyes (e.g. Lysotracker), which preferentially accumulate in acidic vesicles such as late endosomes and lysosomes, with proton sponge-based NPIs could influence the outcome of 
colocalization assays. Since proton sponge-based NPIs exert a buffering effect on endosomes, they could potentially inhibit staining with these acidotropic dyes. However, it is not clear if successful staining of endosomes in combination with proton sponge-based NPIs indicate a lack of buffering effect since an increased flux of protons into the endosome would allow acidification and thus staining of the endosomes [116-118]. It is also possible to use fluorescently labeled ligands (e.g. LacCer, CTB and cholera toxin) with known internalization pathway (Figure 3C) [119]. Nevertheless, it should be noted that even though these markers may exploit distinct uptake mechanisms, further IC trafficking of these markers is rarely differentiated and they frequently end up colocalized [100]. In this regards they are more suited for studying internalization pathways rather than IC trafficking. 
A

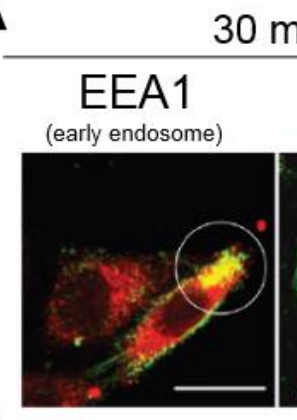

B

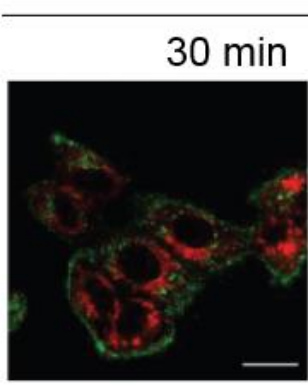

Lysotracker

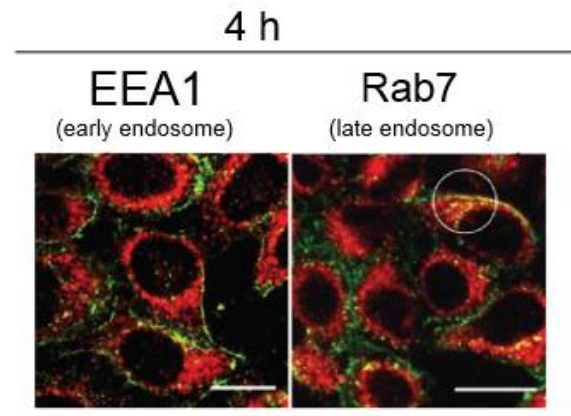

$24 \mathrm{~h}$
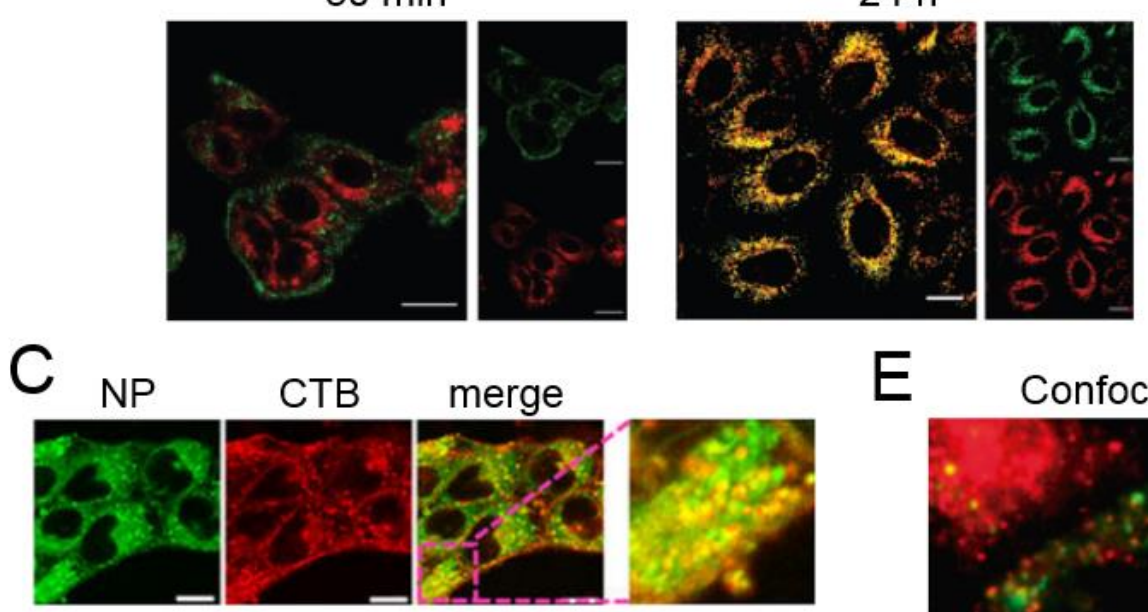

NP

\section{CTB}

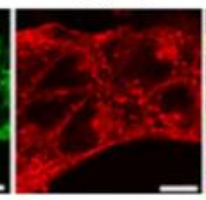

merge
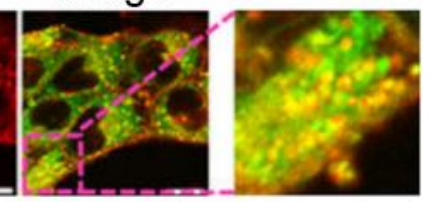

$\mathrm{E}$
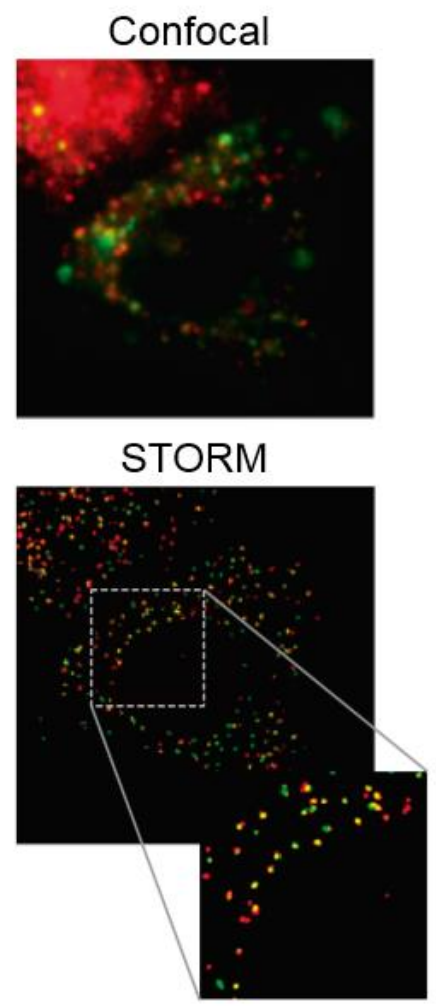

Fig. 3 Evaluation of NPI colocalization with intracellular structures. (A) NPs are labeled in green and are incubated with the cells for $30 \mathrm{~min}$ or $4 \mathrm{~h}$ before staining of specific endocytic proteins using AF568-labeled antibodies (red). Antibodies were used to label the early endocytic marker EEA1 and the late endocytic marker Rab7. Scale bar represents $20 \mu \mathrm{m}$. Images adjusted from [120] with permission of The Royal Society of Chemistry. (B) NPs are labeled in green and are incubated with cells for $30 \mathrm{~min}$ or $24 \mathrm{~h}$ before staining the lysosomes with Lysotracker Red. Scale bar represents $20 \mu \mathrm{m}$. Images adjusted from [120] with permission of The Royal Society of Chemistry. (C) Upper images: cells were incubated for $4 \mathrm{~h}$ with $\mathrm{C} 6$ labeledNPIs (green) and AF594-Cholera toxin subunit B (CTB; red), a known endocytic marker of caveolae-mediated internalization. Lower images: cells are incubated for $4 \mathrm{~h}$ with Cy5 labeled-NPIs (red) and AF488-CTB (green). Scalebar represents $10 \mu \mathrm{m}$. Images adapted from [121]. (C 2017 Public License (D) Spatiotemporal trafficking profile of p(CBA-ABOL) nanoplexes in ARPE19 cells. Graph shows the percentage of polyplexes that colocalize with late endosomal markers (LAMP1, Rab7, Golgi, Flotilin2 
and Rab11), obtained with the dynamic colocalization algorithm based on trajectories. Image reprinted with permission of [108]. (C) 2011 American Chemical Society. (E) Upper image: cell incubated with polystyrene NPs (red) and macropinosomelabeling (green); imaged via conventional confocal microscopy. Lower image: same cell imaged via stochastic optical reconstruction microscopy (STORM), demonstrating the vast improvement in resolution over traditional confocal microscopy. Image size is $40 \mu \mathrm{m}$ and inset size is $5 \mu \mathrm{m}$. image adapted with permission from [26]. (C) 2016 Americal Chemical Society.

It is of note that some types of highly scattering or reflective NPs, such as AuNPs, can be visualized by microscopy in reflectance mode so that they do not have to be labeled with a fluorophore. This only works well using a microscope with optical sectioning ability, such as confocal microscopy or total internal reflection microscopy (TIRM). For instance, TIRM was used to study the colocalization of GFPclathrin with $500 \mathrm{~nm}$ colloidal particles. Unfortunately, since the penetration depth of the evanescent field is limited to approximately $200 \mathrm{~nm}$, TIRM can only be used to study events close to the plasma membrane at the coverslip interface, i.e. the initial stages of NPI internalization [122]. For trafficking in live cells it is important to use a microscope that combines speed with sensitivity. For instance, a disadvantage of CLSM is the relatively low rate at which images can be recorded, especially when visualizing small and/or dim objects which need slow scan speeds to collect enough photons. A solution is provided by spinning disk confocal microscopy, where a disk with a series of pinhole apertures is used so that several pixels are illuminated simultaneously and light can be collected from all of them at the same time. In this way, fast confocal acquisition in live cells can be performed, albeit at a somewhat reduced confocality (i.e. lower contrast) due to pinhole crosstalk [123].

In static colocalization, the overlap of two objects is analyzed based on static images and color merges are frequently used to provide qualitative information (e.g. green + red $=$ yellow). However, a proper understanding of these experiments requires quantitative analysis of colocalization. Strategies for static colocalization quantification can be categorized into object-based or pixel-based, the latter being subdivided into co-occurrence and correlation measures. In co-occurrence methods, the Manders coefficients are well-established and calculate the percentage of total signal from one channel that overlaps with signal from the other channel, based only on the presence or absence of signal. In correlation measures, the Pearson coefficient measures linear correlation, i.e. the extent of a relationship between signals in both channels [124-126]. Besides static colocalization, dynamic 
colocalization, where a colocalization algorithm looks for correlated movements of two objects, can be used as well. In dynamic colocalization, photobleaching is increased because of longer acquisition times but chances for false positives are highly reduced. Moreover, dynamic colocalization allows spatiotemporal characterization of NPI internalization. Vercauteren et al. first performed quantitative live-cell fluorescence microscopy to study the IC processing of poly(amido amine) poly(N,NOcystaminebisa- crylamide 4-aminobutanol) ( $($ (CBA-ABOL)) NPIs in retinal pigment epithelium (RPE) cells. They found that NPIs were specifically delivered to Rab5- and flotillin-2-positive vesicles and were subsequently trafficked to Rab7 and LAMP-1-labeled endolysosomes, where the major fraction remained entrapped (Figure 3D) [108]. When objects are closer to one another than the optical resolution limit, they always appear to be colocalized in static microscopy images. This is resolved in dynamic colocalization experiments where it is the correlated motion of two objects (NPI and endosome) that is taken as a measure of true colocalization. While being more reliable, it is, however, more demanding in terms of experiments and analysis. Also super-resolution techniques are proving useful to enhance the reliability of colocalization experiments. Shang et al. prepared NPs for superresolution imaging and imaged their internalization in living cells with STED nanoscopy [102]. Van der Zwaag et al. evaluated colocalization of $80 \mathrm{~nm}$ PS NPs with the plasma membrane, macropinosomes (Figure 3E), nuclear membrane and actin using STORM, thereby allowing to resolve subdiffraction endocytic vesicles [26]. Li and coworkers used dynamic colocalization via PALM to visualize clathrindependent internalization of polystyrene NPs with different sizes by COS-7 cells [127]. However, the true competence of super-resolution microscopy in the intracellular trafficking of NPIs remains to be demonstrated.

Due to the limitations associated with both exclusion and colocalization assays, it is worth mentioning that these assays are often performed simultaneously to confirm or further investigate observations. A nice example is provided by Sahay et al. who identified the internalization pathway required for cationic siRNA-containing lipid nanoparticle (LNP) entry into HeLa cells. Downregulation of Cdc42 and Rac1 (regulators of macropinocytosis) led to an $80 \%$ decrease in uptake, whereas inhibition of caveolin- 
1 and clathrin heavy chain-1 (regulators of caveolae- and clathrin-mediated endocytosis respectively) had little impact on LNP internalization. Cellular entry through macropinocytosis was then further confirmed through strong colocalization with Cdc42-GFP and ovalbumin-positive vesicles (both markers for macropinocytosis) [17]. Another example is supplied by Zhou et al. who studied the influence of oligoarginine modifications on the internalization of polymeric NPs. The authors performed flow cytometry experiments after treatment with chemical endocytic inhibitors, colocalization assays using immunostaining of specific endocytic proteins (e.g. Rab 7) and colocalization with organelle-specific fluorescent markers (e.g. Lysotracker) [120].

Another method to study the distribution of NPIs in endocytic vesicles is subcellular fractionation, where organelles such as early endosomes, late endosomes and lysosomes are separated through density-gradient centrifugation $[128,129]$. Although fractionation allows evaluation of a large number of cells, the method has proven to be experimentally challenging. The main drawbacks are the difficulty of identifying endocytic vesicles after centrifugation since they have many marker proteins in common and do not vary greatly in density. Furthermore, the requirement of breaking the cells before separating the intact organelles can induce damage or rupture of internal vesicles. Therefore, Lazebnik and coworkers developed an approach based on the polymerization of 3,3'-diaminobenzidine (DAB), a membrane-permeable molecule, by endocytosed horseradish peroxidase (HRP), a marker for fluid phase pinocytosis, in the presence of $\mathrm{H}_{2} \mathrm{O}_{2}$. In this way, only vesicles containing HRP were crosslinked, wich increased the vesicles' density and made the vesicles resistant to detergents. As a consequence, insoluble crosslinked vesicles (early endosomes, late endosomes or lysosomes - determined by localization and thus incubation time of HRP) were easily separated from non-crosslinked vesicles using a detergent. Next, the relative fractions of PEI/AF647-siRNA NPIs in early endosomes, late endosomes and lysosomes were quantified by measuring the fluorescence of the supernatant and comparing it to the fluorescence of the supernatant of matching samples where $\mathrm{H}_{2} \mathrm{O}_{2}$ was omitted and thus crosslinking was inhibited [27]. Alternatively, Bertoli and coworkers used the magnetic properties of NPs to isolate NP-containing endosomes from A549 cells at different times. Recovered fractions were 
tested against different markers of the endolysosomal pathway by Western Blot to evaluate the distribution of NPs [130].

Although used less frequently, analytical methods can be employed to study NPI endocytosis, provided that the NPI meets the rather specific requirements related to the selected method such as magnetic properties or absorbing of the material to a metallic surface for SERS applications. In 2014, Hofmann et al. introduced a mass spectrometry method to study IC trafficking. IC vesicles containing superparamagnetic iron oxide polystyrene NPs (SPIOPSN) were magnetically isolated and their protein composition was analyzed by label-free quantitative MS. Proteomics identified relevant proteins involved in a macropinocytic-like internalization pathway, guiding the NPs via macropinosome-like vesicles towards the final destination inside multilamellar lysosomes [131]. Huefner et al. successfully applied a reporter-free SERS method to generate a color-coded distribution of PCA-LDA (principal component analysis - linear discriminant analysis) derived spectral features in SERS maps of cells, allowing detailed hyperspectral characterization of endosomes and lysosomes in SH-SY5Y human neuroblastoma cells. Furthermore, this method allowed determination of vesicular localization of AuNPs and revealed the molecular environment throughout the cellular uptake pathway [132].

\subsection{Exocytosis}

Cells use the process of exocytosis to remove membrane proteins such as transporters and ion channels, to excrete essential molecules, to communicate with other cells or to repair the plasma membrane [133]. However, this natural cellular process can interfere with the delivery of NPIs for gene therapy. During endocytic confinement, NPIs can be exocytosed via three different routes. First, early endosomes can fuse with recycling endosomes that direct the cargo towards the plasma membrane for recycling. Second, following early endosome maturation into late endosomes, multivesicular bodies (MVBs) can arise that fuse with the plasma membrane, thereby releasing their content outside the cell, for instance in the form of exosomes. Contrary to the lumen of these MVBs that can inherit NPIs from late endosomes, exosomes can contain NPIs only when MVBs pick them up from the cytoplasm after 
successful endosomal escape upon formation of the exosomes. A similar type of extracellular vesicles, named ectosomes, originate directly from budding at the plasma membrane and could also contain NPIs, picked up from the cytoplasm, if endosomal escape was realized [22,134-136]. Thirdly, the ERGolgi secretory pathway, active after intracellular sorting of the NPI cargo, is defined as "conventional exocytosis" [22]. In contrast to the extensive study of cellular uptake mechanisms of NPIs in the last decades, little is known to date about NPI excretion from cells. Understanding the involvement of exocytosis is relevant because exocytosis of NPIs may cause a change in protein corona, leading to unwanted toxicity or unpredicted biological effects [133]. Additionally and most importantly, efficiency of gene delivery is effectively reduced when NPIs are directed back out of the cells $[17,133,137]$.

The most straightforward method to quantify exocytosis of NPIs is to measure the amount of expelled NPs in fresh medium or the decrease of internalized NPs in function of time. One way to do this is by measuring the fluorescence of labeled NPIs [17]. Shukla et al. estimated exocytosis of AF647-labeled siRNA from HSC-T6 cells by evaluating the fluorescence in freshly added medium after several time points [138]. Alternatively, Yanes et al. measured the decrease in IC fluorescence after administration of FITC-labeled mesoporous silica-based nanomedicines to A549 cells as an indication of the amount of exocytosis [139]. Likewise, Jiang et al. studied exocytosis of QDs using spinning disk confocal microscopy to calculate the fraction of exocytosed QDs based on the decrease in IC fluorescence after applying fresh cell medium [60]. However, it should be noted that evaluating exocytosis based on a decrease in IC fluorescence is tricky since there are other mechanism that can lower IC fluorescence (e.g. degradation). When working with AuNPs, fluorescent measurements can be replaced by darkfield microscopy to estimate exocytosis as AuNPs are strong light scatterers [140]. Alternatively, ICPcoupled spectrometry methods can be used for quantitative assessment of exocytosis of metallic and inorganic NPs $[141,142]$. Strobel et al. used ICP-MS to measure the concentration of Ce (related to amount of $\mathrm{CeO}_{2} \mathrm{NPs}$ ) in cell culture medium supernatants of HMEC-1 cells with or without the addition of exocytosis inhibitors [143]. However, with this approach it is not possible to differentiate between intact NPIs and their breakdown products $[133,144]$. 
Fluorescence microscopy can be used as well to study NPI exocytosis. Besides live-cell imaging to observe exocytosis in real-time [145], colocalization with relevant subcellular structures is frequently used as well. To evaluate exocytosis in the form of exosomes, colocalization with CD9, an established protein marker of exosomes, can be studied [105]. However, the most studied protein involved in endocytic recycling is Rab11, a protein that is primarily associated with recycling endosomes and regulates recycling of endocytosed material $[146,147]$. An example is again provided by Sahay et al., who found that LNP showed colocalization with Rab11-positive recycling endosomes, labeled via Rab11-antibodies or by transfection with Rab11-GFP pDNA. Surprisingly, however, Rab11 depletion did not improve LNP retention. Depletion of Rab8a (which blocks secretion from the Golgi/ER after material exchange with late endosomes) and Rab27b (which blocks exosome secretion and/or fusion with the plasma membrane) did increase LNP retention, leading to enhanced silencing. This phenotype of increased retention was also observed when LNPs were applied to mouse embryonic fibroblasts devoid of Niemann Pick type C1 (NPC1). NPC1 is a 13 transmembrane glycoprotein that is located on the surface of late endosomes. Subcellular trafficking of lipids from late endosomes and lysosomes towards the EC environment has been reported to utilize NPC1 and the absence of this protein causes late endosomal and lysosomal dysfunction. Combining all this information led the authors to conclude that LNPs are exocytosed through NPC1-mediated recycling, independent of Rab11 function. This result was consistent with previous reports that indicated that NPC1-mediated recycling was independent of Rab11 function even though some colocalization with Rab11-positive vesicles was shown [17]. Besides colocalization assays, single particle tracking (SPT) can be used to study exocytosis. Jin et al. used SPT to study the movement of single-walled carbon nanotubes as they were expelled from NIH-3T3 cells. Comparable to dynamic colocalization, photobleaching is the major limitation for SPT measurements, since the photobleaching time constrains the total observation time [148].

\subsection{Endosomal escape}

Cellular uptake through the process of endocytosis results in the cargo residing in endosomes, thus being physically separated from the cytoplasm by the endosomal limiting membrane. Escape out of 
the endosomes is generally required since endosomal sequestration potentially causes enzymatic degradation in lysosomes, recycling out of the cell or may prevent the particle or its therapeutic cargo from reaching the intended intracellular target. NPIs are, therefore, often designed to have some form of endosomal escape mechanism $[13,116,149]$. Several approaches have been developed to promote endosomal escape of NPIs including the use of cell penetrating peptides, the flip-flop phenomenon, the proton sponge mechanism, pore formation and membrane fusion. A detailed description of these mechanisms has been subject of numerous reviews $[150,151]$. However, despite extensive research performed in the last decades, endosomal escape remains a highly inefficient process as, typically, less than $10 \%$ of NPI-containing vesicles show efficient endosomal release [152]. Even though it has been proven difficult, quantifying endosomal escape efficiency and elucidating release mechanisms of NPIs could improve our understanding of this formidable IC barrier, which in turn may lead to the design of new and improved NPIs [153]. Besides quantification, qualitative information about the mechanism of endosomal escape can be useful since enhancing endosomal escape efficiency requires prior knowledge about the mechanism of endosomal release. Therefore several assays that provide information about endosomal escape mechanisms are available, as discussed in more detail in a recent review by our group [116].

In the context of NA delivery, endosomal escape efficiency is often related to the extent of reporter protein expression as a consequence of mRNA and pDNA translation or silencing of the reporter protein expression by siRNA $[116,154,155]$. Although endosomal escape is related to the final level of protein expression, one should be careful interpreting these results since several other barriers could influence the therapeutic effect of NAs after successful endosomal escape. As such there is a need for more specific assays to quantify endosomal escape specifically without the influence of subsequent barriers.

The most well-established method to evaluate endosomal escape efficiency is via fluorescence microscopy. The transition from a punctate (endosomal confinement) to a diffuse (cytosolic release) 
intracellular fluorescence pattern (IFP) is frequently used as a qualitative confirmation of endosomal escape [116]. Since out of focus light can falsely give the impression of diffuse staining, confocal microscopy is recommended to evaluate the IFP $[116,153]$. A first way to do this is by co-incubation with small fluorescent molecules such as labeled dextrans or calcein, as shown in Figure 4A [156-159]. Calcein has the specific advantage that it can be loaded in self-quenching concentrations, making the diffuse cytoplasmic staining brighter due to dequenching of the fluorescence upon dilution of calcein into the cytoplasm. However, one should remain cautious when drawing conclusions from these experiments, since the release of a small molecule such as calcein doesn't necessarily guarantee the release of the cargo of interest. Calcein release can be evaluated after co-incubation with a wide array of NPIs but also after incorporation into the lumen of liposomes. When present in the liposomal lumen, calcein will reach the cytosol due to fusion of liposomes with the endosomal membrane, as membrane fusion is the preferential mechanism of endosomal release for liposomes [160,161]. Loading calcein inside the liposome has the benefit of visualizing the NPI itself and avoiding interactions between the NPI and the co-incubation solution. A related methodology to determine the endosomal escape capacity of a variety of NPls was provided by Rehman et al. who co-incorporated a high concentration of fluorescently labeled oligonucleotides (ONs) in NPIs. When the NPI resides in the endosome, the ON fluorescence is mostly quenched. Upon endosomal escape, the labeled ONs are released, resulting in a local burst of fluorescence due to dequenching and eventual accumulation into the nucleus, as illustrated in Figure 4B. With this method it is not only possible to count the number of endosomal escape events within a cell, it also allows to visualize endosomal escape in real-time, pinpointing endosomal escape events in time and place. Additionally, by evaluating the rate of ON accumulation into the nucleus, the authors showed that the polymeric carrier PEI induces a sudden burst (protonsponge effect) whereas a more gradual leaking occurred with a lipid based carrier [162]. The transition of a punctate to a diffuse pattern to evaluate endosomal escape can also be used if the cargo itself is labeled. Clearly, this would only work for cargo that is small enough (e.g. siRNA) to diffuse through the cytoplasm upon endosomal release. Basha et al. used this strategy to examine the effect of different 
lipids on the cytoplasmic delivery of cy5-labeled siRNA [163]. However, the dose needed to visualize cytosolic siRNA with most microscopes is far above the therapeutic range [164]. To extend the dynamic range, Wittrup et al. used a spinning disk microscope with two different exposure settings. Most of the cellular volume was imaged with short exposure times so as to visualize the brightest structures within the cells (e.g. intact lipoplexes and vesicles). Next, they focused on a particular plane in the cells and recorded an image with a long exposure time, intentionally overexposing bright areas to detect weakly fluorescent siRNA signal in the cytosol. Using this method, they could observe the sudden cytosolic release of AF647-siRNA from lipoplex-containing vesicles in HeLa cells, as depicted in Figure 4C. Furthermore, they found that galectin- 8 recruitment can be used to identify the siRNA-releasing endosomes as it recruits to the damaged endosome within 5-10 s after cytosolic siRNA detection [152].

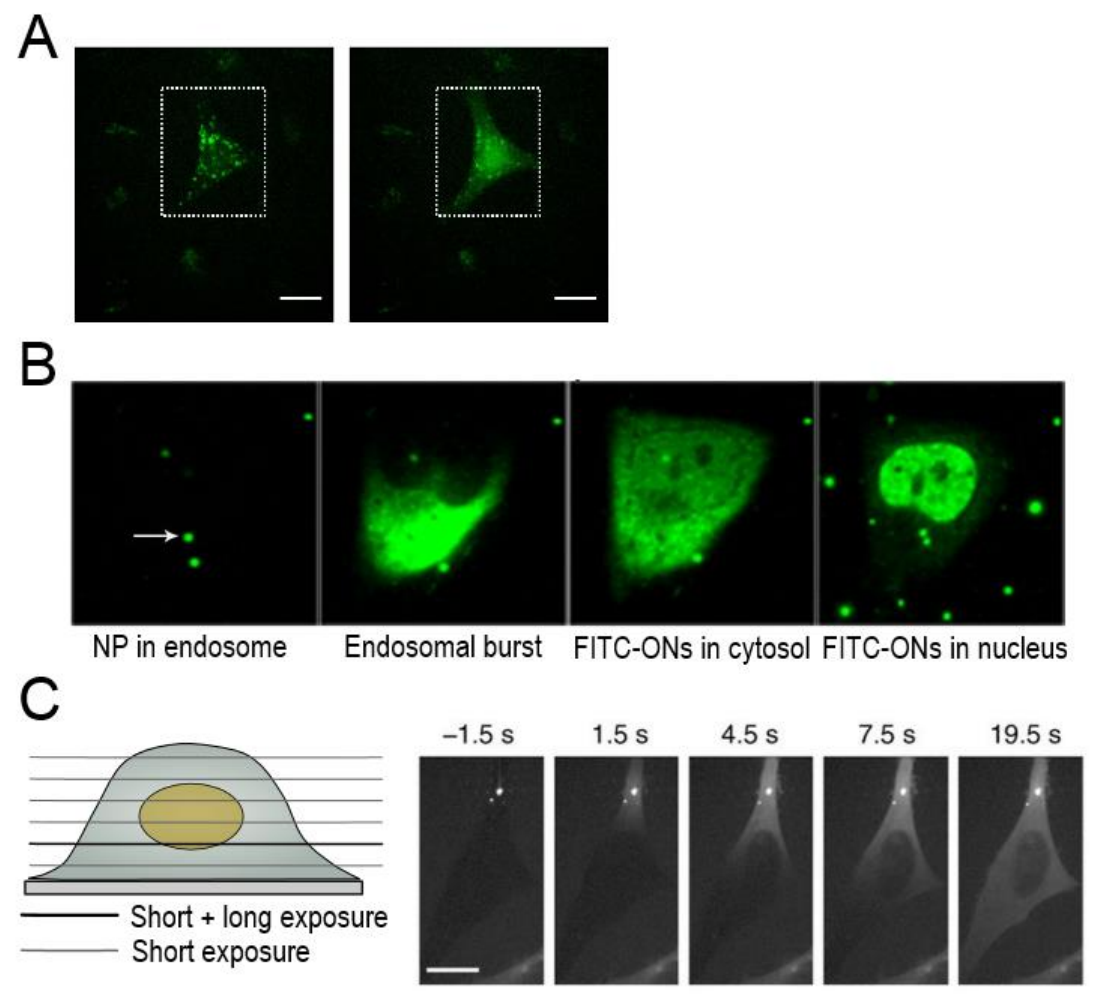

Figure 4 Microscopic evaluation of endosomal escape. (A) Visualization of calcein uptake and release after co-incubation with AuNPs conjugated to cell-penetrating peptides for $4 \mathrm{~h}$. Left: the cell in the dotted box shows a clear punctate intracellular fluorescence pattern (IFP) due to endosomal uptake of calcein. Right: calcein has spread as a diffuse staining towards the entire cytoplasm, indicative of endosomal escape. Scalebars represent $20 \mu \mathrm{m}$. Images adapted [159]. (c) 2016 Public License. (B) Upon endocytosis of the NPI with co-incorporated FITC-labeled ONs, the fluorescence of these ONs is efficiently quenched. The NPI can be seen as a fluorescent dot (white arrow in frame 1). Upon endosomal escape, the fluorescence of these ONs is dequenched (frame 2) and spreads throughout the entire cytoplasm (frame 3). Eventually, FITC-ONs will readily accumulate inside the nucleus (frame 4). Images reprinted with permission of [162]. (C) 2013 American Chemical Society (C) Left: protocol used by Wittrup et al. to visualize sudden cytosolic release of AF647-siRNA. Short exposure times, adjusted to the brightest 
structures in the cell, were used to image the cell at several z-positions (grey lines). Next, a single plane was captured with longer exposure time to detect the weakly fluorescent siRNA signal in the cytosol. Right: single-plane images at long exposure times reveal that the released siRNA rapidly diffuses throughout the entire cytoplasm upon endosomal escape. Scale bar represents $10 \mu \mathrm{m}$. Images reprinted with permission from Springer Nature Nature Biotechnology [152] @ 2015.

In order to distinguish cytosolic NPIs from those still sequestered in endolysosomal compartments, colocalization analysis can be employed. When NPIs are situated in the cytoplasm, they are no longer colocalized with endosomes. Therefore, the lack of colocalization with endosomal compartments is an indirect measure for endosomal escape $[110,165-167]$. Of course this method essentially relies on being able to see all types of endosomes. Non-specific endosomal markers such as dextrans or transferrins or markers that will end up in most endocytic vesicles like plasma-membrane specific dyes are best suited for this.

Changes in the microenvironment of the NPI can also be useful to evaluate endosomal escape efficiency. For instance, Deng et al. developed a ratiometric $\mathrm{pH}$-sensing nanoprobe that was used for quantitative, high-throughput evaluation of endosomal escape. The nanoprobes were co-incubated with several polymeric NPIs in order to examine endosomal escape efficiency of the NPIs. This intracellular $\mathrm{pH}$-sensing probe is able to estimate the efficacy of endosomal escape since it senses a change in $\mathrm{pH}$ when going from the acidic endosomal environment to the cytosol, thereby altering its fluorescence [168]. Alternatively, Wang et al. designed a quantitative redox-activatable sensor (qRAS) for the real-time monitoring of cytosolic delivery of macromolecules, again via co-incubation. The qRAS-labeled macromolecules are silent inside the endosomal compartment but are switched on by redox activation upon delivery into the cytosol after endosomal escape [169]. The main benefit of these methods lies within the fact that they can be used as a high-throughput technique to evaluate endosomal escape. However, the use of high-throughput measurements does not allow for direct visualization of the processes, in contrast to confocal microscopy, a largely qualitative and lowthroughput technique $[168,169]$.

Due to its unparalleled resolution, electron microscopy (EM) allows to visually distinguish between NPls that are sequestered in endolysosomal vesicles or located freely in the cytoplasm $[110,165,170]$. 
However, excessive sample preparation and complex image analysis limit the amount of cells that can be evaluated, thereby making it difficult to extract quantitative information. Furthermore, fixation artefacts can make visual assessment of endosomal escape challenging [116]. Gilleron et al. developed a semi-automatic quantification of EM images where automatic detection of LNP-siRNA-gold NPs was followed by manually assigning the particles to either endosomal compartments or the cytoplasm in order to quantify endosomal escape. This way, they estimated that siRNA escape from endosomes into the cytosol occurred at low efficiency and during a limited window of time [164].

Subcellular fractionation can be used as well to physically separate endosomal vesicles from the cytosol. The amount of NP or cargo present in the vesicle or cytosol fractions can then be measured by classic analytical techniques, such as PCR [171,172] and fluorimetry [129]. However, considering the uncertainty of perfect separation of both fractions it is advised to seek further confirmation with other assays [116,171]. Finally, two additional tests are discussed with the sole aim to obtain more information on the mechanism of endosomal escape. In order to examine the influence of $\mathrm{pH}$ on endosomal escape, the $\mathrm{pH}$-responsiveness of the $\mathrm{NPI}$ or the $\mathrm{pH}$ of the endosomal compartment itself could be altered. To this end, V-type proton-pump ATPase inhibitors such as bafilomycins or concanamycins are frequently used since they block the acidification process altogether [173]. The difference in endosomal escape between the normal scenario and the $\mathrm{pH}$-adjusted scenario is then evaluated using the different assays discussed above. The use of buffering agents such as chloroquine, monensin or ammonium chloride is not recommended since they might enhance endosomal escape due to the proton sponge effect rather than block it [116]. Finally, to study endosomal escape via membrane fusion, the fusion of lipids is usually assayed by dye dilution. A frequently used technique in this regard is Förster resonance energy transfer (FRET) where fluorescent markers consisting of a donor and acceptor pair are present on the vesicle surface. Upon membrane fusion, they are diluted over the increased surface area so that FRET efficiency goes down.[174] Dilution can then be monitored via spectrofluorimetry or fluorescence microscopy. The major limitation of this assay is that it cannot distinguish between lipid fusion and lipid mixing, which is important since lipid mixing does 
not result in cytosolic release of the therapeutic cargo. To this end, a fluorescent content marker can be incorporated into the NPI since the loss of its fluorescence will indicate fusion instead of mixing [116].

\subsection{Autophagy}

Distinct from the endocytic pathway, autophagy is mediated by autophagosomes that have engulfed a portion of the cytoplasm for non-selective degradation. As depicted in Figure 1, during the process of autophagy a phagophore is created and evolves into a double-membraned autophagosome upon sequestration of cytoplasmic material. Next, this autophagosome can fuse with a lysosome, generating an autophagolysosome in a process typically referred to as "autophagy flux". Alternatively, an amphisome can be created when an autophagosome merges with an endosome [175]. Autophagy is an evolutionary conserved process that supports cell preservation through degradation of cytosolic material. Recently, it has been discovered that autophagy has a greater variety of physiological roles than initially expected including cell cycle regulation, tumor suppression, development, anti-aging, etc. However, autophagy poses an additional barrier to efficient gene delivery since it can recapture NPIs after their initial escape to the cytoplasm and can redirect them towards lysosomal degradation $[22,176,177]$. Roberts et al. reported that inhibition of autophagy by knockdown of Atg5 (autophagyrelated protein 5) led to an 8-fold increase in gene expression efficiency, thereby confirming that autophagy can act as a substantial barrier in gene therapy [178]. In order to examine the extent of autophagic activity for a particular NPI and cell type combination, a variety of established methods are already available. In 2016, Klionsky et al. published the third version of a guideline paper to standardize the use and interpretation of autophagy assays $[179,180]$.

TEM was the first method used to evaluate autophagy and still remains important today as it provides highly detailed information. Assuming sufficient expertise, TEM allows detection of the distinct steps of autophagy since autophagy organelles show a specific morphology $[181,182]$. Duan et al. used ultrastructural analysis on TEM images, which indicated that $\mathrm{SiO}_{2} \mathrm{NPs}$ induced autophagy in primary 
human umbilical vein endothelial cells (HUVECs), as shown in Figure 5A-H [183]. Likewise, Lopes et al. found a dose-dependent increase in the autophagic effect of $\mathrm{TiO}_{2}$ NPs on human keratinocytes (HaCaTs) [184]. Furthermore, recent technology breakthroughs in methods used to characterize cellular ultrastructure such as cryo-EM and Focused Ion Beam Scanning Electron Microscopy (FIB-SEM), have only just begun to contribute to autophagy research but promise to increase our understanding of autophagy processes [185].
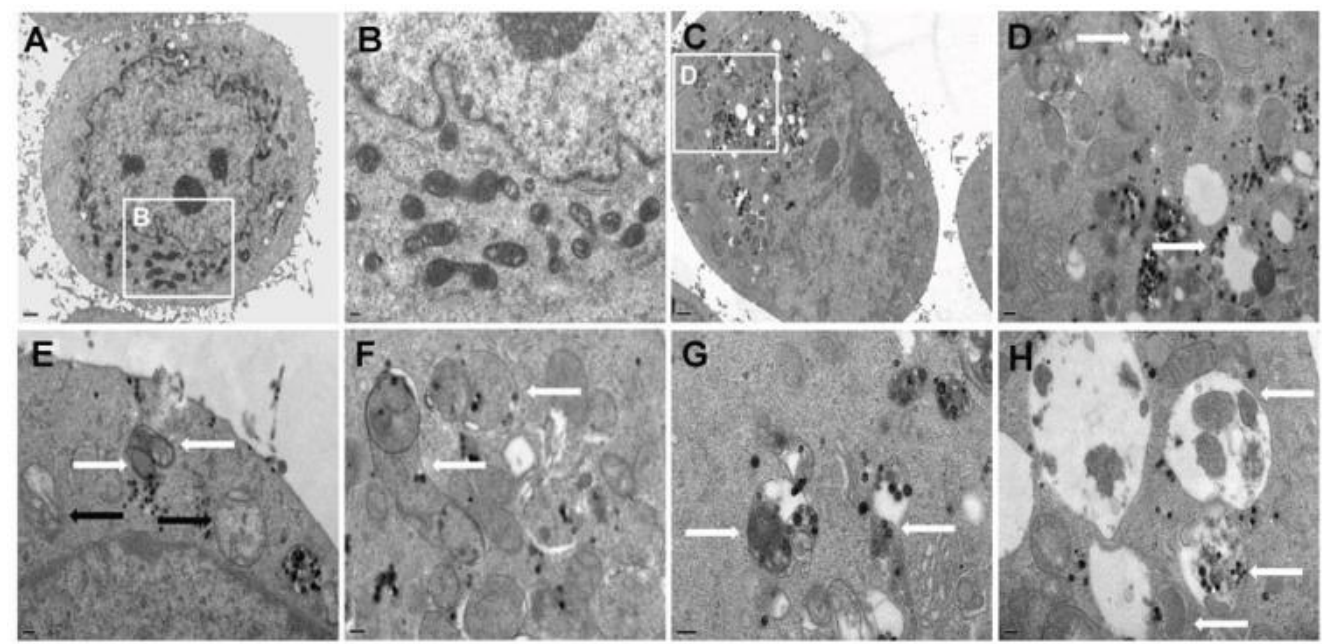

Figure 5 Evaluation of NP-induced autophagy via TEM. (A-B) Untreated human umbilical vein endothelial cells (HUVECs) with normally shaped organelles. (C-D) HUVECs treated with $\mathrm{SiO}_{2}$ NPs show cytoplasmic vacuoles that contain electron-dense NPs (white arrows). (E) Double-membraned phagophores evolving into autophagosomes (white arrows). (F) Autophagosomes (white arrows). (G) Autolyosomes/amphisomes containing cytoplasmic material (white arrows). (H) Autolysosomes/ amphisomes containing mitochondria and $\mathrm{SiO}_{2} \mathrm{NPs}$ (white arrows). Image reprinted with permission from [183].

Using fluorescence microscopy, autophagy is often evaluated by quantifying the number of autophagy-related vesicles. This can be performed using the autophagic dye monodansylcadaverine, which selectively accumulates in autophagic vacuoles presumably because of ion-trapping and/or interaction with membrane lipids $[186,187]$. However, the specificity of this dye remains a matter of debate. Therefore, the more specific microtubule-associated protein 1A/1B light chain 3 (LC3), a major structural protein of autophagosomes, is the most frequently used marker for autophagy. Under normal conditions, cells transfected with a GFP-LC3-encoding construct exhibit a diffuse fluorescence spread throughout the cytoplasm. However, when the number of autophagosomes increases, they can be identified as distinct puncta $[175,181,188]$. Alternatively, colocalization assays of NPIs with autophagy markers such as LC3 are frequently used to evaluate the role of autophagy in NPI-mediated 
gene delivery [17]. However, upon fusion with lysosomes, the autophagosomal membrane is degraded and LC3 expression is lost. Furthermore, LC3 has been observed on other vesicles such as phagosomes and macropinosomes, so it is advised to remain cautious when interpreting results $[108,175,177]$. It must be noted however that above-mentioned assays do not allow differentiation between upregulation of autophagy or an impaired clearance of autophagosomes (inhibition of autophagic flux), since they both result in an increased number of autophagosomes [175]. Co-treatment with lysosomal inhibitors to block lysosomal degradation can be used as an additional test to differentiate between an inhibited autophagic flux or a general induction of autophagy [180]. The distribution of several other proteins related to autophagy, such as p62 (also called sequestosome 1 or SQSTM1), can be evaluated as well. However, the validity of p62 as a marker for autophagy is being questioned since p62 is also involved in other cellular processes $[178,186,189]$.

Alternatively, Western blotting can be used to evaluate autophagy flux or signaling events involved in autophagy. An impaired autophagy flux can be detected by evaluating the ratio of LC3-II/LC3-I (membrane-bound/cytosolic form of LC3) in the presence of lysosomal protease inhibitors (e.g. pepstatin A) or buffers (e.g. chloroquine) that inhibit LC3-II degradation. When LC3-II remains constant in the presence or absence of the inhibitor, autophagy flux is likely to be blocked. GFP-LC3 cleavage can also be determined by Western Blotting in order to evaluate autophagy flux. Since GFP is resistant to lysosomal hydrolysis, the amount of free GFP is correlated to autophagosome degradation. Additionally, an increase in undegraded p62 can indicate an autophagy flux blockage. Also actively studied is the activity of the mammalian target of rapamycin (mTOR) and its interacting proteins via Western Blotting by means of phospho-specific antibodies $[175,181,190]$. Song et al. postulated that it is important to assess involvement of mTOR-dependent autophagy in NP degradation since they described distinct results for mTOR-dependent and mTOR-independent autophagy regulators. For instance, induction of mTOR-dependent autophagy by rapamycin increased siRNA knockdown whereas induction of mTOR-independent autophagy by LiBr diminished siRNA silencing tremendously [191]. 
Finally, enzyme-linked immunosorbent assay (ELISA) can be used to evaluate autophagy and several kits are already available on the market (e.g. p62, Atg5). These assays allow high-throughput quantification of autophagic activity $[192,193]$. Recently, Oh et al. reported on an assay to evaluate autophagy flux using ELISA with two LC3 antibodies. Using this technique, changes in membraneassociated LC3-II levels and the ratio of LC3-II to LC3-I levels were evaluated. The authors demonstrated that this ELISA technique was more sensitive and reliable for the detection of a blocked autophagy flux compared to the most commonly used LC3 turnover assay via Western blot. However, since ELISA is unable to quantify LC3-I and LC3-II levels simultaneously in a single well, detection should be preceded by separation of subcellular compartments [189].

It should be noted that in order to draw correct conclusions it is advised to combine several techniques to evaluate autophagy. Song et al. evaluated the role of autophagy after administration of lipofectamine 2000 or chitosan NPIs in H1299 cells by showing an increased number of MDC-stained vesicles, a significant increase in LC3-II expression via Western Blotting and colocalization of siRNA with LC3-labeled autophagosomes [191]. Another example is provided by Zhong et al. who evaluated autophagy in mouse fibroblasts after transfection with PEI/DNA polyplexes. Polyplex-induced autophagy was confirmed by visualization of autophagosomes via TEM, light microscopic detection of IC LC3-aggregation into distinctive puncta and elevated expression of LC3-II protein, measured by Western Blotting [194].

\subsection{Vector unpacking}

Another issue that should be considered is the kinetics of cargo release from the carrier. The stability of the interaction between carrier and cargo must be optimized since both premature dissociation and overly stable binding can be disadvantageous to the overall transfection efficiency. On the one hand, the vector needs to protect the cargo from enzymatic degradation through efficient complexation. On the other hand, the cargo needs to be dissociated from the vector prior to interaction with the final IC target (e.g. RISC complex for siRNA, translation machinery for mRNA and transcription machinery for 
pDNA) $[12,18,195]$. It has been stated before that there might be a difference in the best unpacking moment between lipoplexes and polyplexes. This is based on the observation that nuclear microinjection of liposome-pDNA complexes inhibited gene expression while PEI polyplexes did not. This suggests that polymers may still dissociate from the cargo inside the nucleus while lipid carriers should dissociate from their cargo before entering the nucleus $[196,197]$. Modulation of vector unpacking properties can be achieved by changing its chemical features, using stimuli-responsive strategies or by inclusion of enzymes, proteins or chemicals [198].

In order to study cargo association with or dissociation from a certain vector, gel electrophoresis is frequently used. When dissociation has happened, the cargo is free to migrate through the gel and form a detectable band. However, while this method allows to study vector unpacking under controlled lab conditions (e.g. in buffers with different $\mathrm{pH}$ ), it cannot be used to study the stability of complexes in the actual cellular environment [199]. This is again where fluorescence microscopy comes in. In order to examine vector unpacking, the fluorescence intensity of the DNA intercalating dye YOYO-1 can be evaluated. When YOYO-1 labeled pDNA is complexed to the carrier, its fluorescence is decreased because of self-quenching. Consequently, when pDNA is released, the YOYO-1 signal increases substantially, which can be visualized in cells with fluorescence microscopy (or alternatively by flow cytometry) [200]. Another approach is based on colocalization of cargo and carrier, each labeled with a different fluorophore [198]. Using this method, Schaffer et al. demonstrated that vector unpacking can indeed be a limiting step for gene expression in large polymer constructs [201]. However, since components must diffuse far away from each other (further than the microscope's resolution) to be detected as separate, colocalization methods do not provide sufficient sensitivity to detect the onset of dissociation. Förster resonance energy transfer (FRET) imaging may be used instead, which probes interactions on the sub $10 \mathrm{~nm}$ length scale. When the NPI is intact there will be high FRET efficiency, which reduces upon vector unpacking $[198,202]$. One should however be careful when using traditional organic fluorophores since they are susceptible to photobleaching and spectral cross-talk due to broad emission bands. A proposed solution is the use of quantum dots (QDs) as 
efficient FRET-donors since they have a broad absorption, narrow emission spectrum and are highly photostable $[195,198,203]$. Ho et al. used QD-mediated FRET to study the distribution and unpacking of individual chitosan NPIs within HEK293 cells. The use of QD-FRET permitted an immediate detection of changes in the nanocomplex state intracellularly [195]. Another fluorescence based technique to study unpacking of complexes is dual color fluorescence fluctuation spectroscopy (dual color FFS). Here, fluctuations in fluorescence intensities are measured that originate from molecules that move in and out of a confocal detection volume. When cargo and carrier are associated, each labeled with its own fluorophore, the fluorescently labeled molecules move simultaneously, causing similar fluorescence time traces in both spectral channels. When dissociated, the molecules will move independently, resulting in a loss of similarity between both signals [198]. Lucas et al. successfully used dual color FFS on rhodamine green-labeled oligonucleotides complexed with Cy5-labeled polymers in order to characterize IC vector unpacking in Vero cells. On the downside, it should be noted that the number of particles that can be analyzed is limited since the detection volume is very small (femtoliters) and particles are highly diluted in the cytosol [204].

Magnetic resonance imaging (MRI) was also reported for the evaluation of vector unpacking. Park et al. conjugated ultrasmall superparamagnetic iron oxide (USPIO) to PEI polymers. This conjugate was capable of complexing pDNA and the $\mathrm{T}_{2}$ relaxation time of water enhanced by USPIO was shown to be a function of the packaging state of the vector. This technique can be used to study vector unpacking in in vivo situations since USPIO NPs itself are used as a contrast agents for MRI imaging with high resolution. Furthermore, NPI unpacking is studied while avoiding fluorescent labeling of NPIs, thereby eliminating the issue of in vivo tissue autofluorescence and the issue of possible alteration of vector unpacking of unlabeled NPIs vs fluorescently labeled NPIs [205].

\subsection{Cytoplasmic degradation}

Following successful endosomal escape and vector unpacking another significant barrier to gene delivery arises: nucleases present in the cytoplasm can degrade released NAs. For instance, several 
studies have demonstrated that the half-life of pDNA in the cytoplasm is in the range of $50-90$ min $[2,5,177]$. Surprisingly, research on NA degradation in the cytosol remains rather limited to date, presumably because only few techniques are available to investigate this experimentally.

In order to investigate the integrity of NAs, gel electrophoresis can be used [206]. However, specificity of this technique is limited to lab conditions since free NAs and other biomolecules will generate a high background in complex biologic environments. Alternatively, PCR can be used to investigate NAs because of the method's high specificity and sensitivity. Recent advances in quantitative PCR have made measurements in undiluted biological samples possible by using a PCR buffer with a higher $\mathrm{pH}$, eliminating the need for DNA isolation [207]. However, besides detecting intact plasmids, PCR also quantifies partially degraded plasmids that contain the amplified region, which is of course undesirable when evaluating NA degradation. A more suitable method to evaluate degradation would be Southern Blotting, where only intact plasmids are detected and quantified, as was performed by Vitiello et al. $[208,209]$.

The above mentioned methods in any case do not allow to study NA degradation in real-time within the cell. An advanced fluorescence microscopy method in this regard is fluorescence correlation spectroscopy (FCS). From the fluctuations measured by this technique, arising from labeled NAs moving through the confocal detection volume, the diffusion coefficient of those molecules in the cell can be obtained from fitting a theoretical model to the experimental autocorrelation function. Using FCS, degradation can be monitored by an increase in diffusional rate of the NA [210]. Sasaki and Kinjo monitored the size and structure of double-labeled DNA by FCS and fluorescence cross-correlation spectroscopy (FCCS) after introduction of the DNA into the cytoplasm of COS7 cells by bead-loading. In FCCS, the fluorescence fluctuation patterns of the two labels are recorded and cross-correlated in order to evaluate if the two labels move simultaneously or not. The authors employed FCS to evaluate the diffusion properties of pDNA and FCCS to monitor the degradation of pDNA at the single molecule level [211]. As FCS and FCCS only obtain information from within a single focal point, Sasaki et al. also 
used cross-correlation raster image correlation spectroscopy (ccRICS) to visualize the spatial distribution of nuclease degradation in live cells. ccRICS was employed to visualize when and where exogenous DNA, injected into the cytosol, is degraded. Their results indicated that nuclease activity in the cytoplasm differs between cell lines, leading to the proposition that nuclease activity impacts the cell's resistance to exogenous DNA incorporation [212]. More accessible than FCS or RICS is confocal FRET imaging, which can be used to determine NA degradation in the cytosol. In this case the NA should be labeled with strategically placed FRET pairs. Upon NA degradation, the FRET pair will dissociate, resulting in lower FRET intensities $[110,213,214]$. Chen et al. developed a two-step QD-FRET approach to monitor vector unpacking and cytoplasmic degradation at the same time. pDNA, double-labeled with QD and a NA dye was complexed with Cy5-labeled polymer. The QD donor drives energy transfer through the intermediate NA dye to the Cy5-labeled polymer. This way, three states of DNA condensation and integrity could be distinguished (complexed and intact, unpacked and intact, unpacked and degraded) [215]. Remaut et al. used FRET-FCS to study the IC degradation of singlestranded oligonucleotides (ONs). ONs were labeled on the $3^{\prime}$ end with rhodamine green and on the $5^{\prime}$ end with Cy5, resulting in high FRET efficiency as long as the ONs are intact and loss of FRET signal when the ON are degraded [216]. Finally, Lechardeur et al. used fluorescence in situ hybridization (FISH) in combination with quantitative single-cell fluorescence video-image analysis to determine for the first time the half-life of single- and double-stranded circular pDNA delivered by microinjection in HeLa and COS cells [217].

\subsection{Nuclear entry}

Nuclear entry is the final barrier that needs to be overcome for successful pDNA delivery [218]. The nuclear envelope consists of an inner and outer nuclear membrane, joined in nuclear pore complexes (NPCs), and the underlying nuclear lamina. NPCs tightly regulate entry into the nucleus as they are the sole channels through which exchange of (macro)molecules happens. Passive entry through the NPCs is limited to molecules less than $10 \mathrm{~nm}$ in diameter, thereby excluding passive pDNA entry $[149,219,220]$. In dividing cells, nuclear entry can occur during mitosis, when the nuclear membrane 
breaks down [221]. However, in non-dividing cells, genetic cargo must cross the nuclear envelope through the NPCs. In order to promote transnuclear transport through the NPCs, the attachment of nuclear localization sequences (NLS) is a traditional strategy. Other methods to enhance nuclear translocation include linking of nuclear proteins or small molecule ligands. One example is the binding of the glucocorticoid receptor ligand dexamethasone to the plasmid. When dexamethasone binds to the glucocorticoid receptor, its NLS is exposed and the entire DNA-ligand-receptor complex can be translocated into the nucleus $[222,223]$. Unfortunately, nuclear entry remains rather poorly studied and requires more extensive research to investigate potential improvements [5].

Nuclear uptake efficacy is indirectly evaluated in most studies simply by measuring the efficiency of gene expression. However, it is necessary to not only measure the final effect of the loaded therapeutic agent but also the amount of delivered cargo to the nucleus [196]. Qualitative evaluation of nuclear uptake can be provided by CLSM $[224,225]$ and other forms of microscopy such as EM [226]. Although these methods give an idea about the presence or absence of nuclear delivery, it is very difficult to relate this information to the actual percentage of cargo (from the total amount administered) that has been efficiently delivered to the nucleus [220].

When quantifying the total amount administered to the nucleus, it is often difficult to differentiate between NPIs residing in the cytoplasm and NPIs residing in the nucleus. Therefore, in order to correctly measure nuclear uptake, the nucleus needs to be separated from other cellular components before the measurement. Here, it is important to make a distinction between nuclear-membrane associated particles and particles that are effectively internalized into the nucleus [220]. Cohen et al. compared a density gradient technique to a detergent-based method to isolate the nuclei of transfected cells and found that the density gradient procedure yielded nuclei with substantially less plasmid attached on the outside of the nuclear membrane. Next, they determined the number of plasmids that reach the nucleus after transfection with PEI- or Lipofectamine-based NPIs using an internally standardized quantitative PCR assay. This method accurately measures the ratio of plasmids 
to a single-copy gene and thus the number of nuclei, thereby directly revealing the amount of plasmids per nucleus [227]. In the quantification of nuclear uptake, PCR is the most widely used method since it is able to quantify plasmid numbers, has good sensitivity and avoids the need to fluorescently label the cargo upon transfection $[208,227,228]$. Besides PCR, Southern blotting was also used to quantify nuclear uptake without the need of using fluorophores [208]. However, efforts are being made to introduce techniques that allow a higher throughput. One example is provided by Bishop et al., who developed a flow cytometry-based assay to quantify the number of plasmids within the nucleus after nuclei isolation. Conversion from fluorescence intensities, measured by flow cytometry, to plasmid numbers was performed through calibration via quantitative PCR and fluorimetry. Fluorimetry was used to measure the fluorescence of several calibration solutions with a known amount of NAs (determined by quantitative PCR). Flow cytometric analysis can provide important information since it is able to quantify nuclear uptake on the single cell level [20]. Alternatively, the amount of metallic NPIs present in the nucleus can be measured by ICP-MS. Huo et al. determined the number of Au-TIOP NPIs in MCF-7 cells via ICP-MS after cell nuclei were extracted [226].

It should be noted however that cellular fractionation and nuclear isolation usually involve centrifugation which could lead to inaccurate conclusions due to precipitation of cell-surface bound and cytoplasmic NPIs within the nuclei pellet [220]. An elegant solution to avoid errors generated by cell fractionation and nuclear isolation protocols was proposed by Tammam et al. who assessed nuclear delivery in intact cells using FRET fluorometry and microscopy. The authors used Hoechst (nuclear staining) as the donor and fluorescein (present on cargo - in this case albumin) as the acceptor of the FRET pair and corrected the measurements for cross talk. When the cargo is successfully delivered to the nucleus, the dyes are located in close proximity from each other and a FRET signal can be measured [229]. However, this assay might be less ideal when using NAs as cargo since Hoechst is commonly used to stain DNA and will highly likely interact with NA-cargo outside the nucleus. 
Table 1. Overview of techniques with their general pros and cons used to visualize and/or quantify specific intracellular barriers.

\begin{tabular}{|c|c|c|c|c|c|c|c|c|c|c|}
\hline \multirow{2}{*}{ Technique } & \multirow{2}{*}{ Advantages } & \multirow{2}{*}{ Limitations } & \multicolumn{8}{|c|}{ Intracellular barriers with references } \\
\hline & & & $\begin{array}{l}\text { Uptake } \\
\text { efficiency }\end{array}$ & $\begin{array}{l}\text { Uptake } \\
\text { pathway }\end{array}$ & Exocytosis & $\begin{array}{l}\text { Endosomal } \\
\text { escape }\end{array}$ & Autophagy & $\begin{array}{c}\text { Vector } \\
\text { unpacking }\end{array}$ & $\begin{array}{l}\text { Cytoplasmic } \\
\text { degradation }\end{array}$ & $\begin{array}{c}\text { Nuclear } \\
\text { entry }\end{array}$ \\
\hline Flow cytometry & $\begin{array}{l}\text { High throughput } \\
\text { Easy to perform } \\
\text { Single cell measurements } \\
\text { (semi)quantitative technique }\end{array}$ & $\begin{array}{l}\text { Requires fluorescent labeling } \\
\text { No spatial information }\end{array}$ & {$[31,32,78]$} & {$[100-106]$} & [139] & {$[168,169]$} & & [200] & & \\
\hline $\begin{array}{l}\text { Fluorescence } \\
\text { microscopy }\end{array}$ & $\begin{array}{l}\text { Easy to perform } \\
\text { Single cell measurements } \\
\text { Direct visualization } \\
\text { Spatio-temporal information }\end{array}$ & $\begin{array}{l}\text { Low throughput } \\
\text { Requires fluorescent labeling } \\
\text { Unable to resolve single particles }\end{array}$ & $\begin{array}{l}{[55-60} \\
73,78]\end{array}$ & $\begin{array}{c}{[103,104,107} \\
108,110,113 \\
114,119]\end{array}$ & $\begin{array}{c}{[17,60,105} \\
139 \\
145-148]\end{array}$ & $\begin{array}{l}{[37,152} \\
164-167 \\
156-163]\end{array}$ & $\begin{array}{l}{[181,183,} \\
184,194]\end{array}$ & $\begin{array}{c}{[195,} \\
200-202, \\
204]\end{array}$ & [211-217] & {$[224,225]$} \\
\hline Electron microscopy & $\begin{array}{l}\text { Single cell measurements } \\
\text { Spatial information } \\
\text { Able to resolve single particles }\end{array}$ & $\begin{array}{l}\text { Very low throughput } \\
\text { Extensive sample preparation } \\
\text { Complex image analysis } \\
\text { No live-cell imaging possible } \\
\text { Limited quantitative information }\end{array}$ & {$[65-73]$} & & & $\begin{array}{l}{[110,164,} \\
165,170]\end{array}$ & $\begin{array}{l}{[17,181} \\
186-188 \\
191,194]\end{array}$ & & & [226] \\
\hline Analytical techniques & $\begin{array}{c}\text { High throughput } \\
\text { (semi)quantitative technique }\end{array}$ & $\begin{array}{l}\text { Population measurement } \\
\text { No spatial information }\end{array}$ & & & & & & & & \\
\hline Fluorimetry & Easy to perform & Requires fluorescent labeling & {$[78]$} & [27] & {$[138]$} & {$[129,169]$} & & & & \\
\hline Mass spectroscopy & Sensitive & Destructive sampling required & [79-82] & [131] & $\begin{array}{c}{[139,} \\
141-143]\end{array}$ & & & & & [226] \\
\hline Blotting & Readily available & $\begin{array}{l}\text { Difficult to perform } \\
\text { Time-consuming }\end{array}$ & & {$[130]$} & & & $\begin{array}{l}{[181,190} \\
191,194]\end{array}$ & & {$[209]$} & [208] \\
\hline ELISA & $\begin{array}{l}\text { Readily available } \\
\text { Easy to perform }\end{array}$ & Time-consuming & & & & & [189] & & & \\
\hline PCR & Sensitive & Time-consuming & [97] & & & {$[171,172]$} & & & [207] & {$[208,227]$} \\
\hline Gel electrophoresis & $\begin{array}{l}\text { Readily available } \\
\text { Easy to perform }\end{array}$ & $\begin{array}{l}\text { Unable to measure sample in } \\
\text { biological environment }\end{array}$ & & & & & & [199] & [206] & \\
\hline
\end{tabular}




\section{CONCLUSIONS}

Non-viral nanoplexes (NPIs) loaded with nucleic acids need to overcome several intracellular (IC) barriers before they can exert their therapeutic function. Detection and quantification of the interaction between NPIs and these IC barriers is crucial to gain a better understanding of these events, which could lead to the rational development of improved NPIs, equipped to conquer the many IC hurdles. In this review, we have given an overview of the methods and assays that are currently available to study the different IC barriers. A summary of the techniques with their representative advantages and limitations is given in Table 1 together with the indication of their applicability to evaluate the different IC barriers. Although there is often a preferred method to study a given barrier, the most reliable and in-depth information is gathered by combining complementary assays. In the future, it might be interesting to gain more detailed information on the interaction between NPIs and their barriers through the use of super-resolution microscopy. However, despite the successful use of super-resolution techniques in biological sciences to image cellular structures [230], their application to probe NPI-cell interactions remains rather limited [102]. Furthermore, efforts should be made in the future to allow investigation of NPI-barrier interactions without the need of labeling, since labeling strategies might alter the behavior of NPls [231]. Therefore, it is again very important to compare results provided by several assays and to think carefully about the advantages and limitation of the methods selected to study the interaction of NPIs and their IC barriers.

\section{ACKNOWLEDGEMENTS}

Financial support of the Agency for Innovation by Science and Technology in Belgium (IWT Flanders) and by the Ghent University Special Research Fund and the Fund for Scientific Research Flanders (FWO, Belgium) is acknowledged with gratitude. This research was funded by the European Research Council (ERC) under the European Union's Horizon 2020 research and innovation program (grant agreement [648214]). The authors have no other relevant affiliations or financial involvement 
with any organization or entity with a financial interest in or financial conflict with the subject matter or materials discussed in the manuscript apart from those disclosed. 


\section{REFERENCES}

[1] X. Guo, L. Huang, Acc Chem Res 45 (2012) 971-979.

[2] L.F. Gottfried, D.A. Dean, Nov. Gene Ther. Approaches (2013) 75-88.

[3] L. Naldini, Nature 526 (2015) 351-360.

[4] D. Ibraheem, A. Elaissari, H. Fessi, Int. J. Pharm. 459 (2014) 70-83.

[5] C.H. Jones, C.-K. Chen, A. Ravikrishnan, S. Rane, B.A. Pfeifer, Mol. Pharm. 10 (2013) 40824098.

[6] H. Yin, R.L. Kanasty, A.A. Eltoukhy, A.J. Vegas, J.R. Dorkin, D.G. Anderson, Nat. Rev. Genet. 15 (2014) 541-555.

[7] M. Ramamoorth, J. Clin. Diagnostic Res. 9 (2015) 1-6.

[8] M. Riley, W. Vermerris, Nanomaterials 7 (2017) 94.

[9] S.L. Hart, Gene Ther. 24 (2017) 121.

[10] U. Lächelt, E. Wagner, Chem. Rev. 115 (2015) 11043-11078.

[11] J. Luten, C.F. van Nostrum, S.C. De Smedt, W.E. Hennink, J. Control. Release 126 (2008) 97110.

[12] L. Li, Y. Wei, C. Gong, J. Biomed. Nanotechnol. 11 (2015) 739-770.

[13] K.L. Kozielski, Y. Rui, J.J. Green, Expert Opin. Drug Deliv. 5247 (2016) 1-13.

[14] A.J. Mellott, M.L. Forrest, M.S. Detamore, Ann. Biomed. Eng. 41 (2013) 446-468.

[15] R. Xiong, S.K. Samal, J. Demeester, A.G. Skirtach, S.C. De Smedt, K. Braeckmans, Adv. Phys. X 1 (2016) 596-620.

[16] I.A. Khalil, K. Kogure, H. Akita, H. Harashima, Pharmacol. Rev. 58 (2006) 32-45.

[17] G. Sahay, W. Querbes, C. Alabi, A. Eltoukhy, S. Sarkar, C. Zurenko, et al. Nat. Biotechnol. 31 (2013) 653-658.

[18] M.P. Stewart, A. Sharei, X. Ding, G. Sahay, R. Langer, K.F. Jensen, Nature 538 (2016) 183-192.

[19] J. Zhou, J.W. Yockman, S.W. Kim, S.E. Kern, Pharm. Res. 24 (2007) 1079-1087.

[20] C.J. Bishop, R.L. Majewski, T.R.M. Guiriba, D.R. Wilson, N.S. Bhise, A. Quinones-Hinojosa, et al. Acta Biomater. 37 (2016) 120-130.

[21] L.Y.T. Chou, K. Ming, W.C.W. Chan, Chem. Soc. Rev. 40 (2011) 233-245.

[22] S. Behzadi, V. Serpooshan, W. Tao, M.A. Hamaly, M.Y. Alkawareek, E.C. Dreaden, et al. Chem. Soc. Rev. 46 (2017) 4218-4244.

[23] N.M. Zaki, N. Tirelli, Expert Opin. Drug Deliv. 7 (2010) 895-913.

[24] G.J. Doherty, H.T. McMahon, Annu. Rev. Biochem. 78 (2009) 857-902.

[25] G. Sahay, D.Y. Alakhova, A. V. Kabanov, J. Control. Release 145 (2010) 182-195.

[26] D. Van Der Zwaag, N. Vanparijs, S. Wijnands, R. De Rycke, B.G. De Geest, L. Albertazzi, ACS Appl. Mater. Interfaces 8 (2016) 6391-6399. 
[27] M. Lazebnik, D.W. Pack, J. Control. Release 247 (2017) 19-27.

[28] A. Elsaesser, A. Taylor, G.S. de Yanés, G. McKerr, E.-M. Kim, E. O’Hare, et al. Nanomedicine 5 (2010) 1447-1457.

[29] B. Drasler, D. Vanhecke, L. Rodriquez-Lorenzo, A. Petri-Fink, B. Rothen-Rutishauser, Nanomedicine (Lond.) 12 (2017) 1095-1099.

[30] S. Palchoudhury, M. Baalousha, J.R. Lead, Front. Nanosci. 8 (2015) 153-181.

[31] A. Guedj, A.J. Kell, M. Barnes, S. Stals, D. Gonçalves, D. Girard, et al. Int. J. Nanomedicine 10 (2015) 5965-5979.

[32] Y. Bai, H. Xing, P. Wu, X. Feng, K. Hwang, J.M. Lee, et al. ACS Nano 9 (2015) 10227-10236.

[33] M. Jaroszeski, G. Radcliff, Mol. Biotechnol. 11 (1999) 37-53.

[34] A. Adan, G. Alizada, Y. Kiraz, Y. Baran, A. Nalbant, Crit. Rev. Biotechnol. 37 (2016) 163-176.

[35] A. Smirnov, M.D. Solga, J. Lannigan, A.K. Criss, J Immunol Methods 423 (2015) 60-69.

[36] A. a Torrano, J. Blechinger, C. Osseforth, C. Argyo, A. Reller, T. Bein, et al. Nanomedicine 8 (2013) 1815-1828.

[37] L.M.P. Vermeulen, T. Brans, S.K. Samal, P. Dubruel, J. Demeester, S.C. De Smedt, et al. ACS Nano 12 (2018) 2332-2345.

[38] H. Liu, A.P.R. Johnston, Angew. Chemie - Int. Ed. 52 (2013) 5744-5748.

[39] G.B. Braun, T. Friman, H.-B. Pang, A. Pallaoro, T.H. de Mendoza, A.-M. a Willmore, et al. Nat. Mater. 13 (2014) 1-19.

[40] E.C. Cho, J. Xie, P.A. Wurm, Y. Xia, Nano Lett. 9 (2009) 1080-1084.

[41] M. Manunta, P.H. Tan, P. Sagoo, K. Kashefi, A.J.T. George, Nucleic Acids Res. 32 (2004) 27302739.

[42] S. Grosse, Y. Aron, G. Thévenot, D. François, M. Monsigny, I. Fajac, J. Gene Med. 7 (2005) $1275-1286$.

[43] P.M. McLendon, D.J. Buckwalter, E.M. Davis, T.M. Reineke, Mol. Pharm. 7 (2010) 1757-1768.

[44] T. dos Santos, J. Varela, I. Lynch, A. Salvati, K.A. Dawson, PLoS One 6 (2011) e24438.

[45] M. Ogris, E. Wagner, P. Steinlein, Biochim. Biophys. Acta 1474 (2000) 237-43.

[46] H.D. Summers, M.R. Brown, M.D. Holton, J.A. Tonkin, N. Hondow, A.P. Brown, et al. ACS Nano 7 (2013) 6129-6137.

[47] R. Ferrari, M. Lupi, F. Falcetta, P. Bigini, K. Paolella, F. Fiordaliso, et al. Nanotechnology 25 (2014) 045102.

[48] M. James, T.D. Giorgio, Mol. Ther. 1 (2000) 339-346.

[49] C. Gottstein, G. Wu, B.J. Wong, J.A. Zasadzinski, ACS Nano 7 (2013) 4933-4945.

[50] K. Remaut, B. Lucas, K. Raemdonck, K. Braeckmans, J. Demeester, S.C. De Smedt, Biomacromolecules 8 (2007) 1333-1340.

[51] K.A. Kang, J. Wang, J.B. Jasinski, S. Achilefu, J. Nanobiotechnology 9 (11) (2011) 16.

[52] K. Rombouts, K. Braeckmans, K. Remaut, Bioconjug. Chem. 27 (2016) 280-297. 
[53] J.L. Kirschman, S. Bhosle, D. Vanover, E.L. Blanchard, K.H. Loomis, C. Zurla, et al. Nucleic Acids Res. 45 (2017).

[54] H. Klingberg, L. B. Oddershede, K. Loeschner, E.H. Larsen, S. Loft, P. Møller, Toxicol. Res. 4 (2015) 655-666.

[55] M. Costanzo, F. Carton, A. Marengo, G. Berlier, B. Stella, S. Arpicco, et al. Eur. J. Histochem. 60 (2016) 107-115.

[56] L. Jiang, X. Li, L. Liu, Q. Zhang, Int. J. Nanomedicine 8 (2013) 1825-1834.

[57] S. Abbad, C. Wang, A.Y. Waddad, J.Z. Huixia Lv, Int. J. Nanomedicine 10 (2015) 305-320.

[58] A.K. Barthel, M. Dass, M. Dröge, J.M. Cramer, D. Baumann, M. Urban, et al. J. Nanotechnol. 5 (2014) 1905-1917.

[59] C.S. Kim, X. Li, Y. Jiang, B. Yan, G.Y. Tonga, M. Ray, et al. MethodsX 2 (2015) 306-315.

[60] X. Jiang, C. Rocker, M. Hafner, S. Brandholt, R.M. Dorlich, G.U. Nienhaus, ACS Nano 4 (2010) 6787-6797.

[61] D. Vanhecke, L. Rodriguez-Lorenzo, M.J.D. Clift, F. Blank, A. Petri-Fink, B. Rothen-Rutishauser, Nanomedicine 9 (2014) 1885-1900.

[62] H. Peuschel, T. Ruckelshausen, C. Cavelius, A. Kraegeloh, Biomed Res. Int. 1 (2015) 1-16.

[63] J.J. Bozzola, L.D. Russell, 2 (1999).

[64] R.F. Egerton, 2 (2016) 1-196.

[65] D. Baiu, N. Artz, M. McElreath, B.D. Menapace, S.B. Reeder, C. Grüttner, Nanomedicine 10 (2015) 2973-2988.

[66] M. Havrdova, K. Polakova, J. Skopalik, M. Vujtek, A. Mokdad, M. Homolkova, et al.Micron 67 (2014) 149-154.

[67] I. George, G. Naudin, S. Boland, S. Mornet, V. Contremoulins, K. Beugnon, et al. Nanoscale 7 (2015) 4529-4544.

[68] M. Kucki, L. Diener, N. Bohmer, C. Hirsch, H.F. Krug, V. Palermo, et al. J. Nanobiotechnology 15 (2017) 1-18.

[69] A.P. Brown, R.M.D. Brydson, N.S. Hondow, J. Phys. Conf. Ser. 522 (2014) 012058.

[70] N. Hondow, R. Brydson, A. Brown, J. Phys. Conf. Ser. 522 (2014) 12055.

[71] N. Hondow, M.R. Brown, T. Starborg, A.G. Monteith, R. Brydson, H.D. Summers, et al. J. Microsc. 261 (2016) 167-176.

[72] E. Guehrs, M. Schneider, C.M. Günther, P. Hessing, K. Heitz, D. Wittke, et al. Nanobiotechnology 15 (2017) 21.

[73] B. Rothen-Rutishauser, D. a Kuhn, Z. Ali, M. Gasser, F. Amin, W.J. Parak, et al. Nanomedicine 9 (2014) 607-621.

[74] F.M. Ross, Science (80-. ). 350 (2015) 9886-1-9886-9.

[75] D.B. Peckys, N. de Jonge, Nano Lett. 11 (2011) 1733-1738.

[76] D.B. Peckys, N. de Jonge, Microsc. Microanal. 20 (2014) 189-197. 
[77] P. Chlanda, J. Krijnse Locker, Biochem. J. 474 (2017) 1041-1053.

[78] M. Claudia, Ö. Kristin, O. Jennifer, R. Eva, F. Eleonore, Toxicology 378 (2017) 25-36.

[79] A.B. Chinen, C.M. Guan, C.H. Ko, C.A. Mirkin, Small 13 (2017) 1-8.

[80] C.T. Ng, F.M.A. Tang, J.J. Li, C. Ong, L.L.Y. Yung, B.H. Bay, Anat. Rec. 298 (2015) 418-427.

[81] T. Schneider, M. Westermann, M. Glei, Arch. Toxicol. 91 (2017) 3517-3527.

[82] J. Saikia, M. Yazdimamaghani, S.P. Hadipour Moghaddam, H. Ghandehari, ACS Appl. Mater. Interfaces 8 (2016) 34820-34832.

[83] Z. Krpetic, S. Saleemi, I.A. Prior, V. See, R. Qureshi, M. Brust, ACS Nano 5 (2011) 5195-5201.

[84] A. Orlando, M. Colombo, D. Prosperi, M. Gregori, A. Panariti, I. Rivolta, et al. J. Nanoparticle Res. 17 (2015) 1-13.

[85] I.-L. Hsiao, F.S. Bierkandt, P. Reichardt, A. Luch, Y.-J. Huang, N. Jakubowski, et al. J. Nanobiotechnology 14 (2016) 1-13.

[86] L.-N. Zheng, M. Wang, B. Wang, H.-Q. Chen, H. Ouyang, Y.-L. Zhao, et al. Talanta 116 (2013) 782-787.

[87] F. Laborda, E. Bolea, J. Jimenez-Lamana, Anal. Chem. 86 (2014) 2270-2278.

[88] L. Amable, C. Stephan, S. Smith, R. Merrifield, PerkinElmer (2017).

[89] S. Hou, K.N. Sikora, R. Tang, Y. Liu, Y.W. Lee, S.T. Kim, et al. ACS Nano 10 (2016) 6731-6736.

[90] L.A. Lane, X. Qian, S. Nie, Chem. Rev. 115 (2015) 10489-10529.

[91] S. Stremersch, M. Marro, B.-E. Pinchasik, P. Baatsen, A. Hendrix, S.C. De Smedt, et al. Small 12 (2016) 3292-3301.

[92] N.M.S. Sirimuthu, C.D. Syme, J.M. Cooper, 82 (2010) 7369-7373.

[93] V. Amendola, M. Meneghetti, S. Fiameni, S. Polizzi, G. Fracasso, A. Boscaini, et al. Anal. Methods 3 (2011) 849.

[94] J. Huang, C. Zong, H. Shen, M. Liu, B. Chen, B. Ren, Z. Zhang, Small 8 (2012) 2577-2584.

[95] X. Shao, P. Schnau, W. Qian, X. Wang, J Nanosci Nanotechnol. 15 (2015) 3834-3838.

[96] P. Jani, G.W. Halbert, J. Langridge, A.T. Florence, J. Pharm. Pharmacol. 42 (1990) 821-826.

[97] S. Colombo, D. Cun, K. Remaut, M. Bunker, J. Zhang, B. Martin-bertelsen, et al. J. Control. Release 201 (2015) 22-31.

[98] E. Carapuça, A.R. Azzoni, D.M.F. Prazeres, G.A. Monteiro, F.J.M. Mergulhão, Mol. Biotechnol. 37 (2007) 120-126.

[99] H. Ragelle, S. Colombo, V. Pourcelle, K. Vanvarenberg, G. Vandermeulen, C. Bouzin, et al. J. Control. Release 211 (2015) 1-9.

[100] D. Vercauteren, J. Rejman, T.F. Martens, J. Demeester, S.C. De Smedt, K. Braeckmans, J. Control. Release 161 (2012) 566-581.

[101] D. Dutta, J.G. Donaldson, Cell. Logist. 2 (2012) 203-208.

[102] L. Shang, P. Gao, H. Wang, R. Popescu, D. Gerthsen, G.U. Nienhaus, Chem. Sci. 8 (2017) 23962400. 
[103] H. Herd, N. Daum, A.T. Jones, H. Huwer, H. Ghandehari, C.M. Lehr, ACS Nano 7 (2013) 19611973.

[104] D. Vercauteren, R.E. Vandenbroucke, A.T. Jones, J. Rejman, J. Demeester, S.C. De Smedt, et al. Mol. Ther. 18 (2010) 561-569.

[105] T. Wang, L. Wang, X. Li, X. Hu, Y. Han, Y. Luo, et al. ACS Appl. Mater. Interfaces 9 (2017) 18619-18625.

[106] A. Benmerah, M. Bayrou, N. Cerf-Bensussan, A. Dautry-Varsat, J. Cell Sci. 112 (1999) 13031311.

[107] C. Rosazza, H. Deschout, A. Buntz, K. Braeckmans, M.-P. Rols, A. Zumbusch, Mol. Ther. Nucleic Acids 5 (2016) e286.

[108] D. Vercauteren, H. Deschout, K. Remaut, J.F.J. Engbersen, A.T. Jones, J. Demeester, et al. ACS Nano 5 (2011) 7874-7884.

[109] P. Watson, A.T. Jones, D.J. Stephens, Adv. Drug Deliv. Rev. 57 (2005) 43-61.

[110] D.J. Lee, E. Kessel, T. Lehto, X. Liu, N. Yoshinaga, K. Padari, et al. Bioconjug. Chem. 28 (2017) 2393-2409.

[111] L. Pelkmans, J. Kartenbeck, a Helenius, Nat. Cell Biol. 3 (2001) 473-483.

[112] A. Hayer, M. Stoeber, D. Ritz, S. Engel, H.H. Meyer, A. Helenius, J. Cell Biol. 191 (2010) 615629.

[113] C.-F. Bassøe, N. Li, K. Ragheb, G. Lawler, J. Sturgis, J.P. Robinson, Cytom. Part B Clin. Cytom. 51B (2003) 21-29.

[114] R. Ragg, F. Natalio, M.N. Tahir, H. Janssen, A. Kashyap, D. Strand, S. et al. ACS Nano 8 (2014) 5182-5189.

[115] J. Wang, S.R. Macewan, A. Chilkoti, Nano Lett. 17 (2017) 1226-1232.

[116] T.F. Martens, K. Remaut, J. Demeester, S.C. De Smedt, K. Braeckmans, Nano Today 9 (2014) 344-364.

[117] I. Richard, M. Thibault, G. De Crescenzo, M.D. Buschmann, M. Lavertu, Biomacromolecules 14 (2013) 1732-1740.

[118] R. V Benjaminsen, M.A. Mattebjerg, J.R. Henriksen, S.M. Moghimi, T.L. Andresen, Mol. Ther. 21 (2013) 149-157.

[119] M.A.E.M. Van Der Aa, U.S. Huth, S.Y. Häfele, R. Schubert, R.S. Oosting, E. Mastrobattista, et al. Pharm. Res. 24 (2007) 1590-1598.

[120] J. Zhou, Y. Chau, Biomater. Sci. 4 (2016) 1462-1472.

[121] L. Zhang, X. Yang, Y. Lv, X. Xin, C. Qin, X. Han, et al. Sci. Rep. 7 (2017) 46186.

[122] G.D. Byrne, D. Vllasaliu, F.H. Falcone, M.G. Somekh, S. Stolnik, Mol. Pharm. 12 (2015) 38623870.

[123] J.-A. Conchello, J.W. Lichtman, Nat. Methods 2 (2005) 920-931.

[124] J.A. Pike, I.B. Styles, J.Z. Rappoport, J.K. Heath, Methods 115 (2017) 42-54.

[125] K.W. Dunn, M.M. Kamocka, J.H. McDonald, Am J Physiol Cell Physiol 300 (2011) 723-742. 
[126] S. Bolte, F.P. Cordelieres, J. Microsc. 224 (2006) 13-232.

[127] Y. Li, L. Shang, G.U. Nienhaus, Nanoscale 8 (2016) 7423-7429.

[128] J. Shi, B. Chou, J.L. Choi, A.L. Ta, S.H. Pun, Mol. Pharm. 10 (2013) 2145-2156.

[129] M. Manunta, L. Izzo, R. Duncan, A.T. Jones, J. Drug Target. 15 (2007) 37-50.

[130] F. Bertoli, G.L. Davies, M.P. Monopoli, M. Moloney, Y.K. Gun'Ko, A. Salvati, et al. Small 10 (2014) 3307-3315.

[131] D. Hofmann, S. Tenzer, M.B. Bannwarth, C. Messerschmidt, ACS Nano 8 (2014) 10077-10088.

[132] A. Huefner, W.L. Kuan, K.H. Müller, J.N. Skepper, R.A. Barker, S. Mahajan, ACS Nano 10 (2016) 307-316.

[133] R. Sakhtianchi, R.F. Minchin, K.B. Lee, A.M. Alkilany, V. Serpooshan, M. Mahmoudi, Adv. Colloid Interface Sci. 201-202 (2013) 18-29.

[134] S. Stremersch, S.C. De Smedt, K. Raemdonck, J. Control. Release 244 (2016) 167-183.

[135] A. Busato, R. Bonafede, P. Bontempi, I. Scambi, L. Schiaffino, D. Benati, et al. Int. J. Nanomedicine 11 (2016) 2481-2490.

[136] D. Ma, Nanoscale 6 (2014) 6415.

[137] N. Oh, J.H. Park, Int. J. Nanomedicine 9 (2014) 51-63.

[138] R.S. Shukla, A. Jain, Z. Zhao, K. Cheng, Nanomedicine Nanotechnology, Biol. Med. 12 (2016) 1323-1334.

[139] R.E. Yanes, D. Tarn, A.A. Hwang, D.P. Ferris, S. Sherman, C.R. Thomas, et al. Small 9 (2013) 697-704.

[140] N. Tlotleng, M.A. Vetten, F.K. Keter, A. Skepu, R. Tshikhudo, M. Gulumian, Cell Biol. Toxicol. 32 (2016) 305-321.

[141] Y. Cui, X. Song, S. Li, B. He, L. Yuan, W. Dai, et al. Oncotarget 8 (2017) 38618-38630.

[142] C.S. Kim, N.D.B. Le, Y. Xing, B. Yan, G.Y. Tonga, C. Kim, et al. Adv. Healthc. Mater. 3 (2014) 1200-1202.

[143] C. Strobel, H. Oehring, R. Herrmann, M. Förster, A. Reller, I. Hilger, J. Nanoparticle Res. 17 (2015) 206-2019.

[144] E. Frohlich, Environ. Toxicol. Pharmacol. 46 (2016) 90-94.

[145] I.I. Slowing, J.L. Vivero-Escoto, Y. Zhao, K. Kandel, C. Peeraphatdit, B.G. Trewyn, et al. Small 7 (2011) 1526-1532.

[146] S. Takahashi, K. Kubo, S. Waguri, A. Yabashi, H.-W. Shin, Y. Katoh, et al. J. Cell Sci. 125 (2012) 4049-4057.

[147] P. Sandin, L.W. Fitzpatrick, J.C. Simpson, K.A. Dawson, ACS Nano 6 (2012) 1513-1521.

[148] H. Jin, D.A. Heller, M.S. Strano, Nano Lett. 8 (2008) 1577-1585.

[149] E.M. McErlean, C.M. McCrudden, H.O. McCarthy, Ther. Deliv. 7 (2016) 619-637.

[150] H.K. Shete, R.H. Prabhu, V.B. Patravale, J. Nanosci. Nanotechnol. 14 (2014) 460-474.

[151] A.K. Varkouhi, M. Scholte, G. Storm, H.J. Haisma, J. Control. Release 151 (2011) 220-228. 
[152] A. Wittrup, A. Ai, X. Liu, P. Hamar, R. Trifonova, K. Charisse, et al. Nat. Biotechnol. 33 (2015) 870-876.

[153] L.I. Selby, C.M. Cortez-Jugo, G.K. Such, A.P.R. Johnston, Wiley Interdiscip. Rev. Nanomedicine Nanobiotechnology 9 (2017) 1-23.

[154] D. Habrant, P. Peuziat, T. Colombani, L. Dallet, J. Gehin, E. Goudeau, et al. J. Med. Chem. 59 (2016) 3046-3062.

[155] A.M. Funhoff, C.F. van Nostrum, G.A. Koning, N.M.E. Schuurmans-Nieuwenbroek, D.J.A. Crommelin, W.E. Hennink, Biomacromolecules 5 (2004) 32-39.

[156] N. Kongkatigumjorn, C. Cortez-Jugo, E. Czuba, A.S.M. Wong, R.Y. Hodgetts, A.P.R. Johnston, et al. Macromol. Biosci. 17 (2017) 1-11.

[157] F. Salomone, F. Cardarelli, M. Di Luca, C. Boccardi, R. Nifosì, G. Bardi, et al. J. Control. Release 163 (2012) 293-303.

[158] V. Hornung, F. Bauernfeind, A. Halle, E.O. Samstad, H. Kono, K.L. Rock, et al. Nat. Immunol. 9 (2008) 847-856.

[159] J. Krawinkel, U. Richter, M.L. Torres-Mapa, M. Westermann, L. Gamrad, C. Rehbock, S. et al. J. Nanobiotechnology 14 (2016) 2.

[160] K. Kono, T. Igawa, T. Takagishi, Biochim. Biophys. Acta - Biomembr. 1325 (1997) 143-154.

[161] S. Itakura, S. Hama, T. Ohgita, K. Kogure, PLoS One 9 (2014) 1-11.

[162] Z.U. Rehman, D. Hoekstra, I.S. Zuhorn, ACS Nano 7 (2013) 3767-3777.

[163] G. Basha, T.I. Novobrantseva, N. Rosin, Y.Y.C. Tam, I.M. Hafez, M.K. Wong, et al. Mol. Ther. 19 (2011) 2186-2200.

[164] J. Gilleron, W. Querbes, A. Zeigerer, A. Borodovsky, G. Marsico, U. Schubert, K. et al. Nat. Biotechnol. 31 (2013) 638-646.

[165] Z. Chu, K. Miu, P. Lung, S. Zhang, S. Zhao, H.-C. Chang, G. Lin, Q. Li, Sci. Rep. 5 (2015) 11661.

[166] Y. Sakurai, H. Hatakeyama, Y. Sato, H. Akita, K. Takayama, S. Kobayashi, et al. Biomaterials 32 (2011) 5733-5742.

[167] H. Akita, K. Kogure, R. Moriguchi, Y. Nakamura, T. Higashi, T. Nakamura, et al. J. Control. Release 149 (2011) 58-64.

[168] Z.J. Deng, S.W. Morton, D.K. Bonner, L. Gu, H. Ow, P.T. Hammond, Biomaterials 51 (2015) 250-256.

[169] Z. Wang, M. Luo, C. Mao, Q. Wei, T. Zhao, Y. Li, G. Huang, J. Gao, Angew. Chemie - Int. Ed. 56 (2017) 1319-1323.

[170] K. Boeneman, J.B. Delehanty, J.B. Blanco-Canosa, K. Susumu, M.H. Stewart, E. Oh, et al. ACS Nano 7 (2013) 3778-3796.

[171] H. Akita, R. Ito, I.A. Khalil, S. Futaki, H. Harashima, Mol. Ther. 9 (2004) 443-451.

[172] S. Hama, H. Akita, R. Ito, H. Mizuguchi, T. Hayakawa, H. Harashima, Mol. Ther. 13 (2006) 786794.

[173] S. Dröse, K. Altendorf, J. Exp. Biol. 200 (1997) 1-8.

[174] A. El-Sayed, I.A. Khalil, K. Kogure, S. Futaki, H. Harashima, J. Biol. Chem. 283 (2008) 23450- 
23461.

[175] K. Peynshaert, B.B. Manshian, F. Joris, K. Braeckmans, S.C. De Smedt, J. Demeester, et al. Chem. Rev. 114 (2014) 7581-7609.

[176] N. Mizushima, Genes Dev. 21 (2007) 2861-2873.

[177] K. Remaut, V. Oorschot, K. Braeckmans, J. Klumperman, S.C. De Smedt, J. Control. Release 195 (2014) 29-36.

[178] R. Roberts, W.T. Al-Jamal, M. Whelband, P. Thomas, M. Jefferson, J. Van Den Bossche, et al. Autophagy 9 (2013) 667-682.

[179] D.J. Klionsky, K. Abdelmohsen, A. Abe, M.J. Abedin, H. Abeliovich, A.A. Arozena, et al. Autophagy 12 (2016) 1-222.

[180] J. Geng, D.J. Klionsky, Autophagy 13 (2017) 639-641.

[181] Q. Wu, R. Jin, T. Feng, L. Liu, L. Yang, Y. Tao, et al. Int. J. Nanomedicine 12 (2017) 3993-4005.

[182] P. Ylä-Anttila, H. Vihinen, E. Jokitalo, E.L. Eskelinen, Methods Enzymol. 451 (2009) 143-164.

[183] J. Duan, Y. Yu, Y. Yu, Y. Li, J. Wang, W. Geng, et al. Int. J. Nanomedicine 9 (2014) 5131-5141.

[184] V.R. Lopes, V. Loitto, J.-N. Audinot, N. Bayat, A.C. Gutleb, S. Cristobal, J. Nanobiotechnology 14 (2016) 22.

[185] J.H. Hurley, E. Nogales, Curr. Opin. Struct. Biol. 41 (2016) 211-216.

[186] N. Man, Y. Chen, F. Zheng, W. Zhou, L.P. Wen, Autophagy 6 (2010) 449-454.

[187] A. Biederbick, H.. Kern, H.. Elsasser, Eur. J. Cell Biol. 66 (1995) 3-14.

[188] J.M. Bravo-San Pedro, F. Pietrocola, V. Sica, V. Izzo, A. Sauvat, O. Kepp, et al. Methods Enzymol. 587 (2017) 71-86.

[189] S. hee Oh, Y. bok Choi, J. hyun Kim, C.C. Weihl, J. sun Ju, Anal. Biochem. 530 (2017) 57-67.

[190] D. Huang, H. Zhou, J. Gao, Sci. Rep. 5 (2015) 14361.

[191] W. Song, Z. Ma, Y. Zhang, C. Yang, Acta Biomater. 58 (2017) 196-204.

[192] Enzolifesciences, (n.d.).

[193] Cusabio, (n.d.).

[194] X. Zhong, D. Panus, W. Ji, C. Wang, Mol. Pharm. 12 (2015) 932-940.

[195] Y.P. Ho, H.H. Chen, K.W. Leong, T.H. Wang, J. Control. Release 116 (2006) 83-89.

[196] R. Tachibana, H. Harashima, Y. Shinohara, H. Kiwada, Adv. Drug Deliv. Rev. 52 (2001) 219-226.

[197] H. Pollard, J.S. Remy, G. Loussouarn, S. Demolombe, J.P. Behr, D. Escande, J. Biol. Chem. 273 (1998) 7507-7511.

[198] C.L. Grigsby, K.W. Leong, J. R. Soc. Interface 7 (2010) S67-S82.

[199] K. Buyens, B. Lucas, K. Raemdonck, K. Braeckmans, J. Vercammen, J. Hendrix, et al. J. Control. Release 126 (2008) 67-76.

[200] V. Zaric, D. Weltin, P. Erbacher, J. Remy, J. Behr, D. Stephan, J Gene Med 6 (2004) 176-184.

[201] D. V. Schaffer, N.A. Fidelman, N. Dan, D.A. Lauffenburger, Biotechnol. Bioeng. 67 (2000) 598- 
606.

[202] G.A. Trusov, A. V Ulasov, E.A. Beletkaia, Y. V Khramtsov, M.O. Durymanov, A.A. Rosenkranz, et al. Biochem. Biophys. 437 (2011) 77-9.

[203] H.H. Chen, Y.-P. Ho, X. Jiang, H.-Q. Mao, T.-H. Wang, K.W. Leong, Mol. Ther. 16 (2008) 324332.

[204] B. Lucas, K. Remaut, N.N. Sanders, K. Braeckmans, S.C. De Smedt, J. Demeester, Biochemistry 44 (2005) 9905-9912.

[205] I.K. Park, C.P. Ng, J. Wang, B. Chu, C. Yuan, S. Zhang, et al. Biomaterials 29 (2008) 724-732.

[206] P. Magadala, M. Amiji, AAPS J. 10 (2008) 565-576.

[207] Y. Bu, H. Huang, G. Zhou, Anal. Biochem. 375 (2008) 370-372.

[208] R. Tachibana, H. Harashima, N. Ide, S. Ukitsu, Y. Ohta, N. Suzuki, et al. Pharm. Res. 19 (2002) 377-381.

[209] L. Vitiello, K. Bockhold, P.B. Joshi, R.G. Worton, Gene Ther. 5 (1998) 1306-1313.

[210] N.L. Thompson, in: Top. Fluoresc. Spectrosc., 2002, pp. 337-378.

[211] A. Sasaki, M. Kinjo, J. Control. Release 143 (2010) 104-111.

[212] A. Sasaki, J. Yamamoto, T. Jin, M. Kinjo, Sci. Rep. 5 (2015) 14428.

[213] M. Hirsch, M. Helm, Nucleic Acids Res. 43 (2015) 4650-4660.

[214] H. Uchiyama, K. Hirano, M. Kashiwasake-Jibu, K. Taira, J. Biol. Chem. 271 (1996) 380-384.

[215] H.H. Chen, Y.-P. Ho, X. Jiang, H.-Q. Mao, T.-H. Wang, K.W. Leong, Nano Today 4 (2009) 125134.

[216] K. Remaut, B. Lucas, K. Braeckmans, N.N. Sanders, S.C. De Smedt, J. Demeester, J. Control. Release 103 (2005) 259-271.

[217] D. Lechardeur, K.J. Sohn, M. Haardt, P.B. Joshi, M. Monck, R.W. Graham, et al. Gene Ther. 6 (1999) 482-497.

[218] J. Nguyen, F.C. Szoka, Acc. Chem. Res. 45 (2012) 1153-1162.

[219] E.E. Vaughan, J. V DeGiulio, D. a Dean, Curr. Gene Ther. 6 (2006) 671-681.

[220] S.N. Tammam, H.M.E. Azzazy, A. Lamprecht, J. Control. Release 229 (2016) 140-153.

[221] N. Symens, S.J. Soenen, J. Rejman, K. Braeckmans, S.C. De Smedt, K. Remaut, Adv. Drug Deliv. Rev. 64 (2012) 78-94.

[222] D. Dean, D. Strong, W. Zimmer, Gene Ther. 12 (2005) 881-890.

[223] A. Lam, D. DA, Gene Ther. 17 (2010) 439-447.

[224] Y. Xu, W. Liang, Y. Qiu, M. Cespi, G.F. Palmieri, A.J. Mason, J.K.W. Lam, Mol. Pharm. 13 (2016) 3141-3152.

[225] K. Chen, L. Guo, J. Zhang, Q. Chen, K. Wang, C. Li, et al. Acta Biomater. 48 (2017) 215-226.

[226] S. Huo, S. Jin, X. Ma, X. Xue, K. Yang, A. Kumar, et al. ACS Nano 8 (2014) 5852-5862.

[227] R.N. Cohen, M.A.E.M. van der Aa, N. Macaraeg, A.P. Lee, F.C. Szoka, J. Control. Release 135 
(2009) 166-174.

[228] R. Moriguchi, K. Kogure, A. Iwasa, H. Akita, H. Harashima, J. Control. Release 110 (2006) 605609.

[229] S.N. Tammam, H.M.E. Azzazy, H.G. Breitinger, A. Lamprecht, Mol. Pharm. 12 (2015) 42774289.

[230] S.J. Sahl, S.W. Hell, S. Jakobs, Nat. Rev. Mol. Cell Biol. 11 (2017) 685-701.

[231] K. Rombouts, T.F. Martens, E. Zagato, J. Demeester, S.C. De Smedt, K. Braeckmans, et al. Mol. Pharm. 11 (2014) 1359-1368. 